\title{
Quantitative streamlines tractography: methods and inter-subject normalisation
}

\author{
Robert E. Smith (a,b), David Raffelt (a), J-Donald Tournier (c), Alan Connelly (a,b,d)
}

October 2, 2020

\begin{abstract}
Recent developments in semi-global tractogram optimisation algorithms have opened the field of diffusion MRI to the possibility of performing quantitative assessment of structural fibre connectivity. The proper application of these methods in neuroscience research has however been limited by a lack of awareness, understanding, or appreciation for the consequences of these methods; furthermore, particular steps necessary to use these tools in an appropriate manner to fully exploit their quantitative properties have not yet been described. This article therefore serves three purposes: to increase awareness of the fact that there are existing tools that attempt to address the well-known non-quantitative nature of streamlines counts; to illustrate why these algorithms work the way they do to give quantitative results; and to explain how to properly utilise these results for quantitative tractography analysis across subjects.
\end{abstract}

\section{Author affiliations}

a The Florey Institute of Neuroscience and Mental Health, Heidelberg, Victoria, Australia

b Florey Department of Neuroscience and Mental Health, University of Melbourne, Melbourne, Victoria, Australia

c Centre for the Developing Brain, School of Biomedical Engineering \& Imaging Sciences, King's College London, London, UK

d Department of Medicine, Austin Health and Northern Health, University of Melbourne, Melbourne, Victoria, Australia.

Corresponding author : Robert E Smith, Florey Institute of Neuroscience and Mental Health, Melbourne Brain Centre, 245 Burgundy Street, Heidelberg, Victoria 3084, Australia, Phone: (+61 3) 9035 7128, Fax: (+61 3) 9035 7301, Email: robert.smith@florey.edu.au

\section{Abbreviations}

AFD: Apparent Fibre Density;

COMMIT: Convex Optimisation Modeling for Microstructure-Informed Tractography;

FBA: Fixel-Based Analysis;

FBC: Fibre Bundle Capacity (an estimate of the bandwidth of a white matter pathway);

FC: Fibre Cross-section (NB: macroscopic change in);

FD: Fibre Density (microscopic);

FDC: Fibre Density and Cross-section (combined measure of FD and FC);

"fixel": Specific fibre population within a voxel;

LiFE: Linear Fascicle Evaluation;

SIFT: Spherical-deconvolution Informed Filtering of Tractograms. 


\section{Introduction}

Since the introduction of tractography to the field of diffusion MRI, there has been extensive interest in use of this technology to assess fibre connectivity in the brain for various neuroscientific applications[1-4]. The vast majority of tractography algorithms operate on the same fundamental mechanism: the "streamlines" algorithm, where plausible white matter fibre pathways are constructed by iteratively propagating along the local estimated fibre orientation[5-8]. Unfortunately, this mechanism of reconstruction does not directly facilitate one of the most fundamental parameters of interest: the density of connectivity between two brain regions[9]. A major contributing factor to this limitation is that while the streamlines algorithm enforces that the reconstructed trajectories obey the estimated orientations of the underlying fibre bundles, it provides no meaningful control over the reconstructed densities of those bundles.

The class of "global tractography" methods[10-14] has for many years shown promise to circumvent this problem. While in the "streamlines" algorithm individual white matter trajectories are propagated independently and using only local fibre orientation information, these "global" methods simultaneously solve for all connections at once, in a manner that enforces the entire tractogram reconstruction to be consistent with the raw diffusion image data. Even the most modern of these methods however incur considerable computational expense (particularly as reconstructions with greater numbers of connections are sought), and typically do not provide any guarantees regarding the construction of connections with biologically meaningful terminations, for instance resulting in terminations in the white matter or CSF which are otherwise considered erroenous[15,16].

A new class of "semi-global" tractogram optimisation algorithms offers a potential compromise[17-21]; these have additionally been referred to as "tractogram filtering", "microstructure-informed tractography", and "bottom-up" algorithms in various contexts. These approaches take as input a whole-brain tractogram generated using one or more streamlines tractography algorithms and modify the reconstruction in some way such that the local streamlines densities become consistent with the density of underlying fibres evidenced by the image data. These methods therefore enable quantitative assessment of fibre connectivity (within the myriad other associated limitations of diffusion MRI and streamlines tractography), with whole-brain reconstructions that are sufficiently dense to enable higher-level analyses (e.g. connectomics) within reasonable computational requirements.

Despite the potential influence of these methods on the neuroimaging field, they have had only limited uptake. This may be due to a lack of awareness of the public availability of such methods, or a lack of understanding that these methods address some of the origins of the limitations of raw streamline count as a metric of connectivity. Furthermore, although these methods seek to modulate the relative connection densities of different white matter pathways within a single brain, the appropriate mechanism by which these quantities should be compared across subjects has not yet been comprehensively explained in the literature. This article therefore serves three purposes, with the aim of increasing the utility of these tools in the field:

- Alert a wider audience to the fact that a primary contributing factor to the non-quantitative nature of streamlines counts can be addressed using freely available methods;

- Carefully explain and demonstrate why the design of these methods is appropriate to provide estimates of white matter connection density;

- Explain how these connectivity estimates should be handled when performing direct comparisons between subjects.

\section{Background}

Before addressing the major points of this article, we first clarify the specific position and role of these "semi-global" tractography optimisation algorithms, the connectivity metric of interest to be derived from them, and the limitations within which they operate.

\section{Context and role of semi-global algorithms}

$\underline{\text { Figure } 1}$ presents the role of these methods within a tractography-based reconstruction pipeline. 


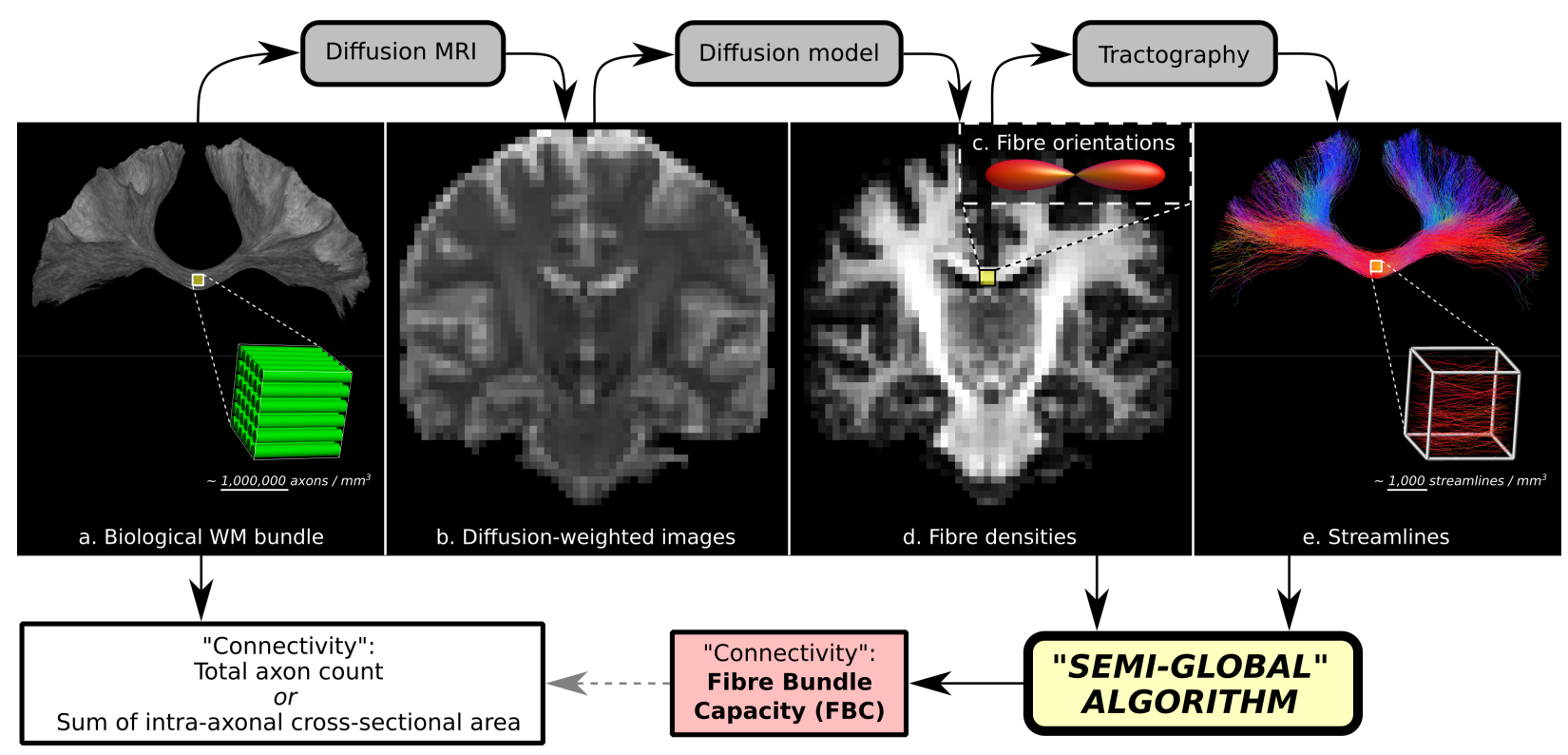

Figure 1: Contextualisation of semi-global tractogram optimisation algorithms. Given the existence of a biological white matter pathway of interest (a), diffusion-weighted imaging is performed (b). A diffusion model is fitted to these data to yield fibre orientation (c) and density (d) estimates. Fibre orientations are utilised by a tractography algorithm to produce streamlines (e). An optimisation algorithm operates on both the tractogram reconstruction and fibre density information to yield an estimate of bundle connectivity, here named "Fibre Bundle Capacity (FBC)", which should ideally be proportional to the connectivity of the biological bundle.

- Some biological white matter bundle of interest (Figure 1a; the connection between homologous motor areas in this example) is interrogated using diffusion-weighted imaging (Figure 1b). Due to the sizes of the underlying axons within the white matter relative to the imaging resolution, there will typically be of the order of a million axons traversing any given image voxel.

The "connectivity" of the biological pathway may be quantified as the number of axons within the connection, or alternatively as the total intra-axonal cross-sectional area, which is directly proportional in the case of fixed axon diameter but also has favourable quantitative properties in the presence of axon diameter variance[22] (more in "Metric of "connectivity"”, section below).

- A diffusion model estimates from these data, within each image voxel, the orientations and densities of the fibre bundles within that voxel (Figure 1c-d).

- These orientation estimates are used by a streamlines tractography algorithm to attempt to reconstruct in a piecewise fashion the fibre within the pathway of interest (Figure 1e). Unlike biological axons, reconstructed streamlines have no associated volume, and are therefore shown as infinitesimally thin in Figure 1e. The number of streamlines traversing any given image voxel may be of the order of 1,000, but varies wildly depending on reconstruction parameters.

Importantly, the raw number of streamlines reconstructed for the pathway of interest should not be interpreted in a quantitative fashion, due to the many user-defined parameters that influence this measure 10.

- The role of such a "semi-global" tractogram optimisation algorithm is to combine the reconstructed tractogram with fibre density information from the diffusion model (or alternatively the diffusion-weighted image data themselves; see "Comparing tractograms and image data" section), relying on the quantitative nature of these fibre density estimates to overcome the non-quantitative nature of streamlines tractography.

- The outcome of such a process is a derived measure of "connectivity" of the pathway of interest (here named "Fibre Bundle Capacity (FBC)"; more in "Metric of "connectivity"" section below). If calculated appropriately, this measure should be a reasonable proxy for the connection strength of the biological pathway. 


\section{Metric of "connectivity"}

The notion of "connectivity" in the context of diffusion MRI tractography remains ambiguous without a very explicit description of exactly what metric is derived from the image data / tractogram reconstruction. In the context of the methods discussed here, we consider the total fibre cross-sectional area of a fibre bundle (ideally, the intra-axonal cross-sectional area) to be our target metric of interest when quantifying "white matter connectivity", for several reasons:

- The sensitivity \& specificity of the diffusion MRI signal (under certain conditions) to the local intra-axonal volume of fibre bundles[23], making this measure readily accessible even from retrospective diffusion MRI data;

- The biological interpretability of such an estimate as a proxy for the "bandwidth" of a connection, i.e. the capacity of a fibre bundle to transfer information[22];

- Other biological parameters that may influence a subjective notion of white matter "connectivity", such as axon diameters and degree of myelination, are not considered here due to the relative insensitivity of conventionallyacquired diffusion-weighted image data to such properties[24,25];

- This quantity is not proportional to the length of a pathway; although bundle length may alter axon conduction delays[26], we consider this to be an independent property of the connection that does not directly influence bandwidth.

To facilitate discussion of higher-level concepts in the context of this metric, we henceforth refer to this metric as the "Fibre Bundle Capacity (FBC)". This term communicates that the intent of this measure is the capacity of a white matter fibre bundle to transmit information between its endpoints. Ideally this measure should be as sensitive and specific as possible to the intra-axonal cross-sectional area of the biological fibres constituting the pathway being reconstructed.

The important distinction between fibre density estimates quantified at the voxel level, and fibre connection density estimates (i.e. FBC) quantified at the level of pathways of interest, is demonstrated in Figure 2. The coloured strips on either side of the image mimic a parcellation of the brain grey matter, with white matter connections between them shown as thick lines. A specific pathway of interest is selected based on those connections identified between the green parcel on the left, and the magenta parcel on the right; the connections ascribed to that pathway of interest are the darker of these lines. As highlighted by the exemplary voxels in Figure 2a-d, there are voxels that include lesser or greater fibre intra-axonal volume ( $\mathrm{a} \& \mathrm{~b}$ and $\mathrm{c} \& \mathrm{~d}$ respectively), and a lesser or greater amount of this may be attributed to the pathway of interest (b\&d and a\&c respectively); no single exemplary voxel provides a quantitative measure that reflects the connectivity of the entire pathway. In contrast, the FBC measure is based on the total fibre intra-axonal cross-sectional area of the entire pathway, as shown at Figure 2e-f. Importantly, this quantity is independent of the location along the length of the bundle at which the measurement is made (assuming no gross ensemble changes in axon diameters along that length), even if the macroscopic cross-section of the bundle does change along this length (as indicated by blue lines at Figure 2e-f).

\section{Limitations of semi-global optimisation algorithms}

- We do not consider the sub-voxel spatial configurations of fibre bundles in either the image[27] or tractogram[28] domains; we consider only that each voxel is the sum of its constituent parts, irrespective of sub-voxel position;

- We assume that the diffusion signal measured in a voxel is the sum of signal contributions from the white matter fibre bundles and other tissues within that volume (i.e. the "slow exchange" regime);

- We do not consider influencing streamlines trajectories based on microstructural information, as discussed[29] and proposed[30] recently; we consider only the use of microstructural / image information to modulate the reconstructed densities of different white matter pathways. 


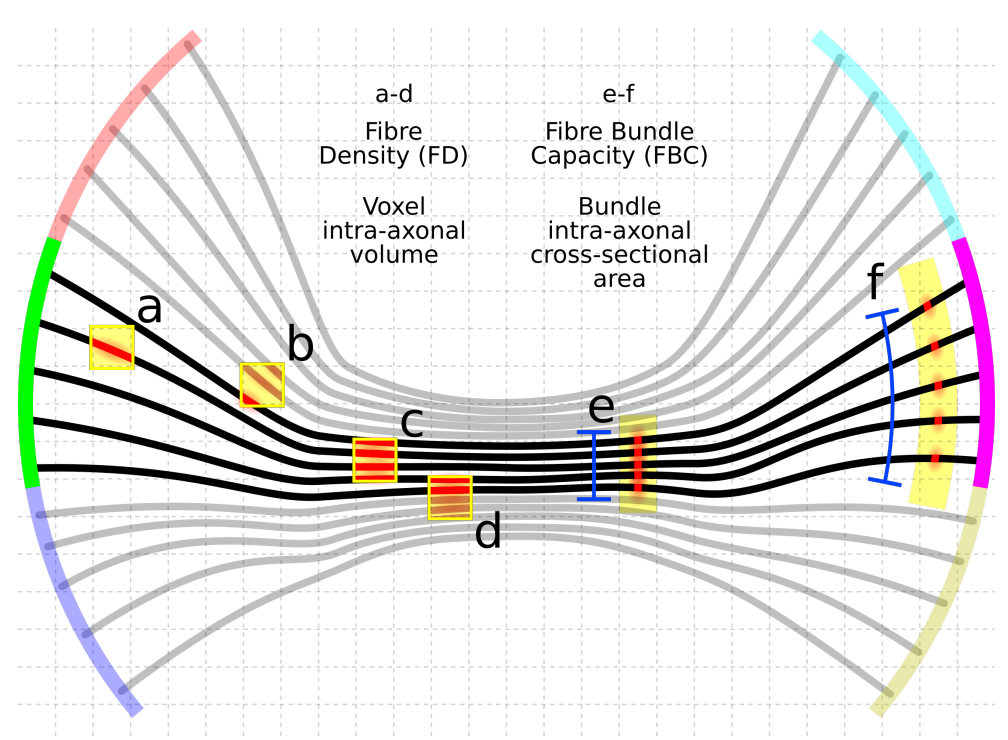

Figure 2: Relationship between quantification of intra-axonal volume within individual voxels (Fibre Density (FD)), and quantification of intra-axonal cross-sectional area of a white matter bundle of interest (Fibre Bundle Capacity (FBC)). A bundle of interest is defined based on selection of two parcels representing the bundle endpoints (green on left side, magenta on right side); connections beloinging to this bundle are shown as darker lines. The Fibre Density (FD) measure is quantified in each individual voxel, based on the intra-axonal volume within that voxel (red highlights in a-d). The Fibre Bundle Capacity (FBC) measure is quantified for the bundle of interest, based on the total intra-axonal cross-sectional area of the connections within the pathway (red highlights in e-f); blue lines demonstrate macroscopic cross-sectional area of bundle.

\section{Comparing tractograms and image data}

Throughout this discussion, we make use of the term "fixel" [31] to refer to a specific fibre population within a particular voxel. Each voxel in the diffusion image may contain multiple fixels ("crossing fibres"), and the number of fixels may vary between different voxels. Use of such terminology assists in disambiguating this concept from a macroscopic white matter fascicle that connects two areas of grey matter, each of which will be associated with many fixels along its length and breadth.

Using diffusion image data to provide a tractogram with quantitative attributes requires a mechanism by which to compare the tractogram to those image data. There are two principal mechanisms by which this may be done (demonstrated in Figure 3):

1. Each streamline contributes some intensity to the reconstructed diffusion signal based on a forward model. Typically, a diffusion tensor with fixed diffusivities is chosen. Signal intensity from sources other than WM fibres may additionally be modelled as isotropic or anisotropic sources contributing to the diffusion signal and included in the optimization. The reconstructed image data from the tractogram ( $\&$ possibly other tissue sources) is compared directly to the empirical diffusion data.

2. The WM fibre density within each fixel is first estimated based on an inverse model, potentially with estimation and separation of other signal sources (e.g. other tissues or fluid)[32-40]. Here we focus on the spherical deconvolution model[41-43], though other approaches can certainly be used. The reconstructed streamlines density from the tractogram ascribed to each fixel is compared directly to the corresponding WM fibre densities estimated from the diffusion model.

Although the former approach is more "conventional", and additionally has a long history of use in the context of global tractography methods, for demonstration purposes we use the latter model, as it provides a more intuitive course of reasoning in the following sections. Note that these two approaches are directly related via the invertibility of the spherical convolution transform: the a priori definition of the forward models to be used for each tissue component / compartment in approach a. serves the same purpose as the a priori definition of the tissue "response functions" for spherical deconvolution in approach b. (Figure 3). Note also that Figure 1 shows case b. only for clarity. 

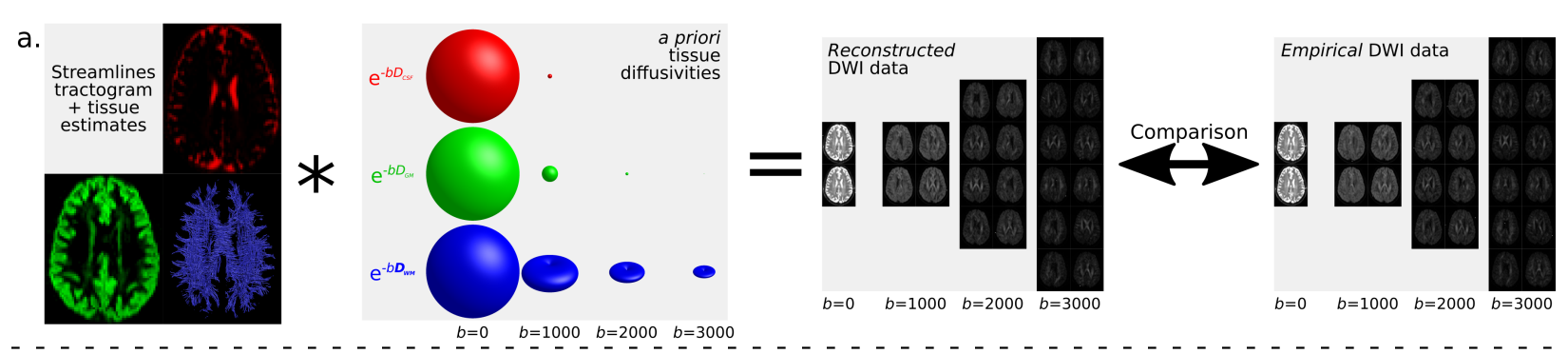

b.
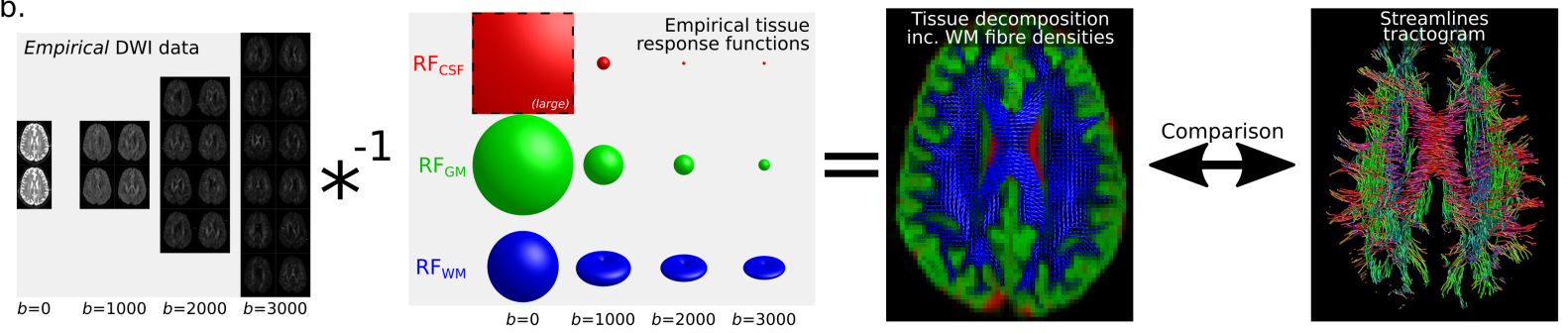

Figure 3: Relationship between two different modelling approaches used in "semi-global" tractography optimisation algorithms. a. Based on estimates of local densities of different types of tissue (including orientation information in the case of WM streamlines), and functions describing how each tissue contributes to the diffusion signal at different $b$-values (often derived from e.g. the diffusion tensor model), a spherical convolution is performed to estimate the diffusion signal from the current tractogram reconstruction. This is compared to the empirical diffusion signal intensity data, and the tissue density estimates within the reconstruction are revised accordingly. b. Based on tissue response functions describing the appearance of each type of tissue in the diffusion data (either from some model, or determined from the image data directly), a spherical deconvolution is performed to obtain estimates of tissue densities (including orientation information in the case of WM fibres). The densities of a reconstructed tractogram are compared to the WM fibre density estimates, and the parameters of the tractogram revised accordingly.

\section{Methods}

\section{The algorithmic basis of quantitative streamlines tractography}

In order to demonstrate the fundamental operation of the algorithms under discussion (and hence the quantitative properties they provide), we begin with a simple definition of the fundamental data and research question that may be applicable to an example analysis involving diffusion MRI tractography.

- What we want:

- An estimate of the Fibre Bundle Capacity (FBC) metric for some pathway (as defined in the "Metric of "connectivity"" section).

- What we have:

- A measure of fibre volume for each fixel as estimated via the diffusion model;

- A set of streamlines delineating the trajectory of the pathway of interest, typically based on a priori regions of interest or other criteria to isolate the pathway;

- A whole-brain tractogram, of which the set of streamlines ascribed to the pathway of interest is a subset (while this is not required for Algorithm 1 below, its necessity will be demonstrated in later algorithms).

The example to be used for demonstration in this article is the connection between left and right precentral sulci, as derived from the "Desikan-Killiany" parcellation[44] provided by the FreeSurfer software[45]; this is shown in Figure 4. Note that while this particular example is shown for only a single pathway of interest, construction of the brain "structural connectome"[46,47] can be thought of as performing this same process for all possible pairs of grey matter regions within a parcellation; hence the ideas presented in this article are applicable in that context also.

We now demonstrate in this section several plausible algorithmic approaches by which our goal may be achieved. We start by proposing a relatively simple and naïve algorithm, observing its benefits and shortcomings, and then use these observations to derive increasingly advanced approaches, eventually presenting a total of four algorithms. 


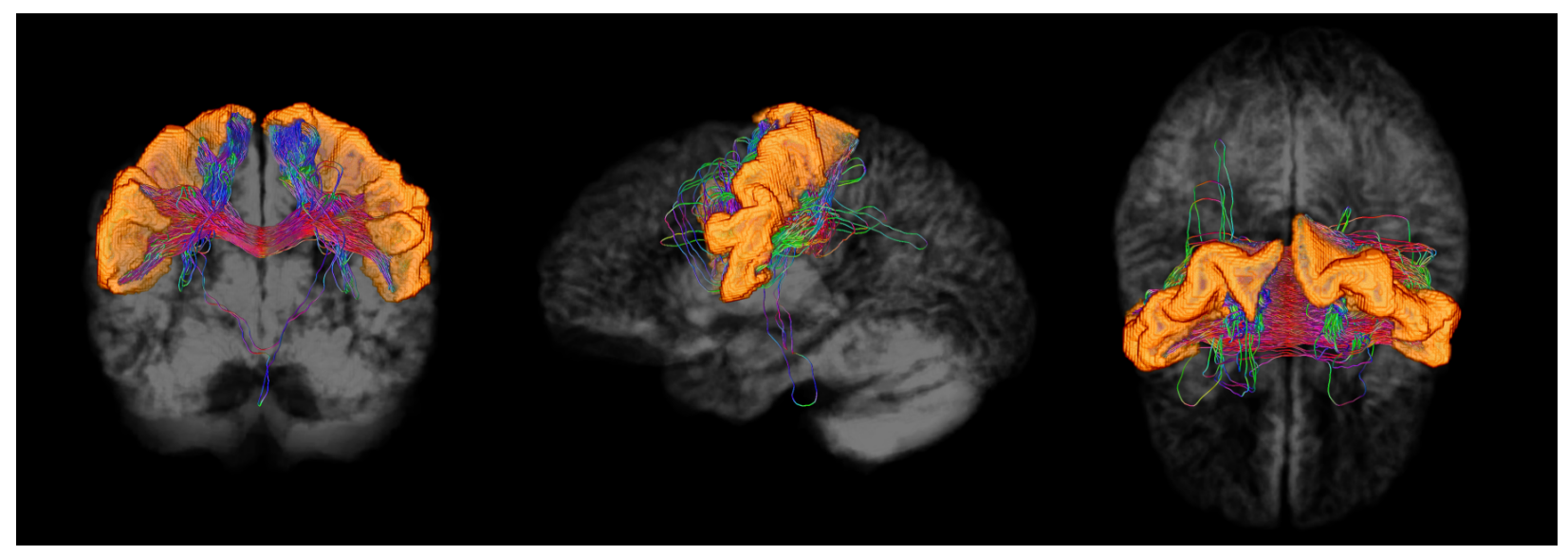

Figure 4: Visualisation of the pathway of interest to be used in demonstration of the four Fibre Bundle Capacity (FBC) quantification algorithms presented in the Methods section. Three orthogonal views, from left to right: coronal, sagittal, axial. The left and right precentral sulci are highlighted in orange; streamlines are coloured according to their local tangent orientation (red = left-right; green $=$ anterior-posterior; blue $=$ inferior-superior).

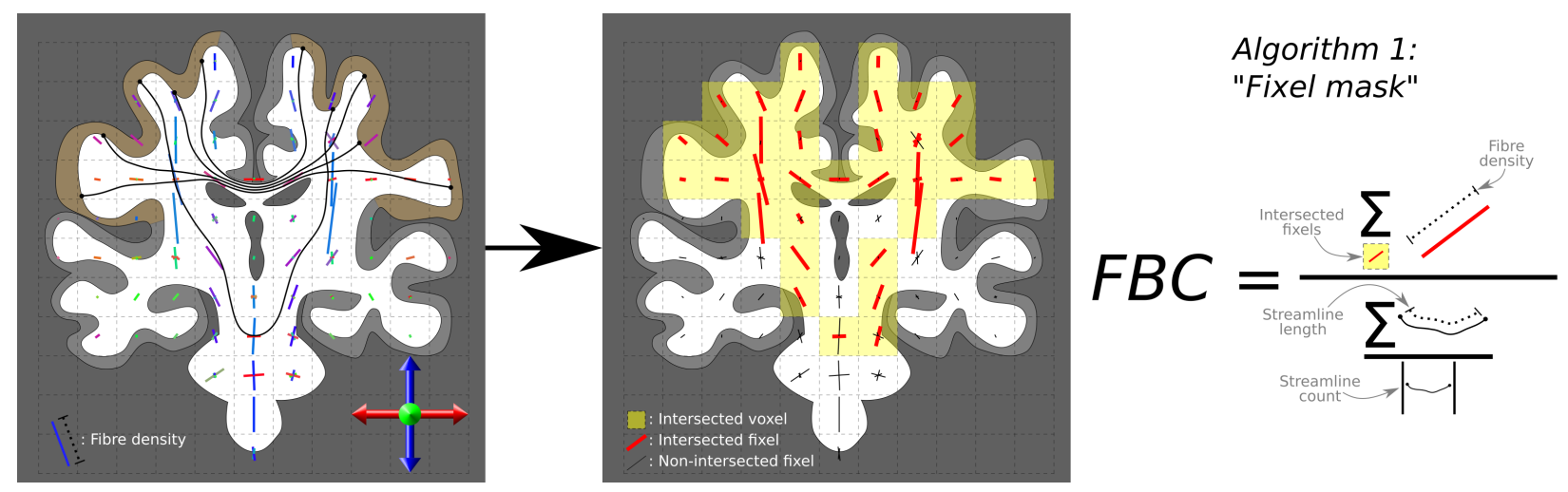

Figure 5: Visual demonstration of the operation of Algorithm 1 ("Fixel mask"). For a particular pathway of interest (left panel: grey matter regions labelled orange, leading to selection of streamlines shown), those fixels traversed by the streamlines corresponding to that pathway are selected (right panel: red fixels within yellow voxels). The sum of the microscopic fibre densities of these selected fixels (equation numerator; encoded visually as fixel lengths) is divided by the mean streamline length (equation denominator: sum of streamline lengths divided by the number of streamlines) to yield the Fibre Bundle Capacity (FBC) measure.

\section{Algorithm 1: "Fixel mask"}

Algorithm 1 is the simplest possible approach for incorporating the fibre density information from a diffusion model into estimating FBC for a pathway of interest reconstructed using streamlines tractography. It is based on the observation that a pathway volume can be derived by computing a mask of fixels that are traversed by the streamlines belonging to that pathway, and this can be converted to a pathway cross-sectional area based on the length of the pathway (Figure 5; also pseudocode provided in Appendix 1):

1. Identify all fixels that are traversed by at least one streamline belonging to the pathway.

2. Sum the fibre volumes of all selected fixels to calculate the total fibre volume of the pathway.

3. Divide this value by the mean streamline length to estimate FBC for the pathway.

Figure 6a shows the assignment of fibre density from the underlying diffusion model field to the quantification of volume (and hence cross-sectional area) of this specific pathway. There are a couple of weaknesses in this algorithm observed in Figure 5 and Figure 6a: 


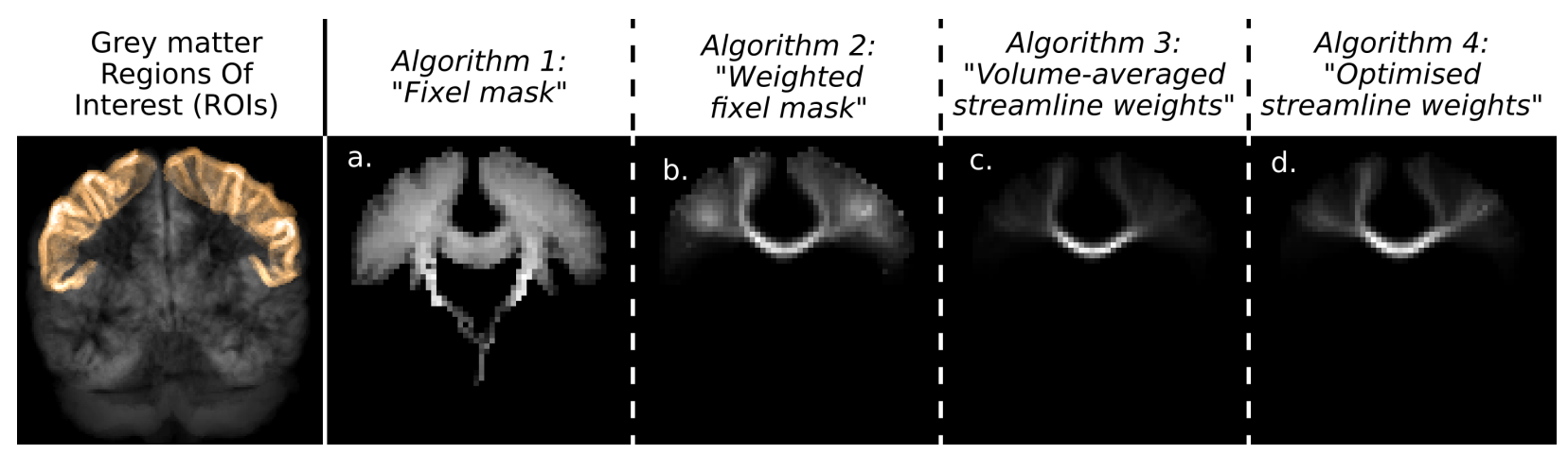

Figure 6: (Left) Coronal projection of brain grey matter, with regions of interest used in reconstruction of the pathway of interest highlighted; (Right) Maximum Intensity Projection (MIP) spatial distributions of the density of white matter fibres attributed to the pathway of interest resulting from quantification using the four algorithms described in the Methods section. The reconstructed bundle is that shown in Figure 4.

- The effect of "outlier streamlines": those streamlines that are attributed to the pathway of interest but follow a trajectory drastically different from the rest of the streamlines assigned to the pathway. When this occurs, all of the fixels traversed by that streamline are added to the fixel mask, and all of the fibre density within each of those fixels contributes to the total fibre volume of the pathway. One or a small number of stray streamlines may therefore drastically increase the final quantification of FBC (e.g. the streamline in Figure 5 that travels partway down the corticospinal tracts as it traverses between the two regions of interest). Utilisation of some more stringent criteria for inclusion of fixels in the mask (e.g. an increased streamline count threshold) could theoretically mitigate this effect, though what form such criteria should take is subjective. Alternatively, such errors may be addressed using additional tractography regions-of-interest or manual quality control procedures; but such mitigation does not trivially extend to studies where many different pathways are assessed (e.g. when building the structural connectome over the whole brain).

- The local fibre density per voxel attributed to the pathway is relatively consistent throughout the entire pathway, from the corpus callosum through the centrum semiovale and to the interface between grey and white matter. This is however contrary to how such tracts are constructed physically: as white matter fibres fan out from the narrow cross-section of the corpus callosum to a long strip of grey matter, the local voxel-wise density of the fibres within this specific pathway would be expected to decrease.

The way in which these effects can manifest, as well as the source of the limitation, is demonstrated in Figure 7. Here the selection of two bundles of interest from a larger white matter pathway is shown, both in the corresponding streamlines and in the fixels to which they are ascribed. What is highlighted in red is the fact that if each of the two bundles are independently mapped to the corresponding voxels traversed, then for the set of voxels intersected by both bundles, all of the fibre density within those voxels will be attributed to both bundles. This has two effects: firstly, the spatial distribution of fibre density attributed to each bundle individually does not vary smoothly, failing to represent partial volume at the outer edge of each bundle similarly to that observed in Figure 6a; secondly, the sum of the calculated fibre connectivity of the two bundles would be greater than that of the actual white matter structure without even necessitating tractography reconstruction errors - since the fibre density in those voxels would contribute to the quantification of both bundles. 

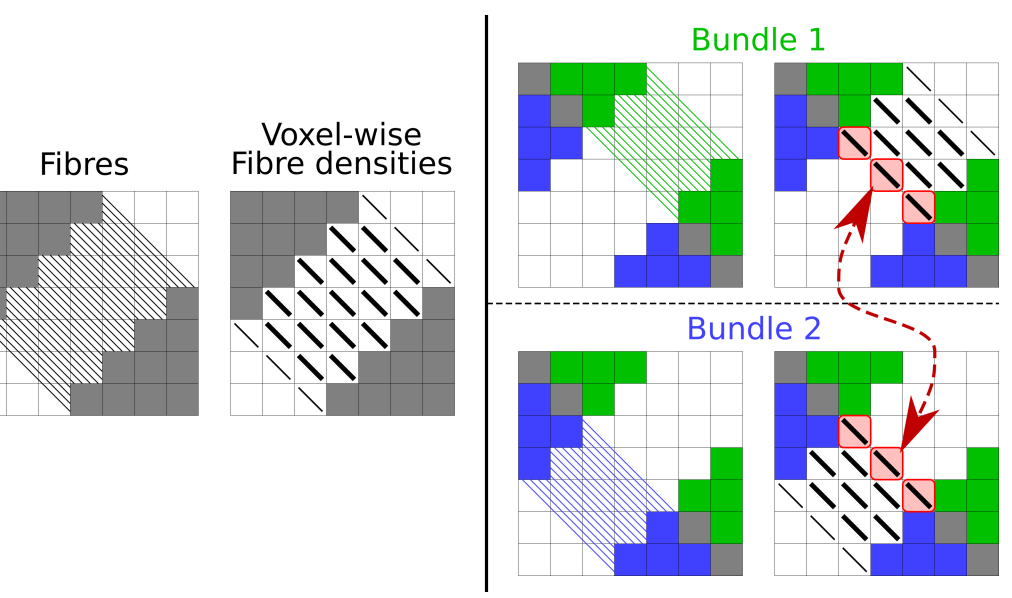

Figure 7: The effects of partial volume on Algorithm 1. Left: A white matter fibre pathway connecting between two grey matter regions, shown as both fibre trajectories and per-voxel fibre orientation / density. Right: The pathway is split into two bundles of interest based on parcellation of the voxels at the endpoints of the pathway; a subset of voxels (highlighted red) are intersected by both bundles.

\section{Algorithm 2: "Weighted fixel mask"}

Algorithm 2 directly addresses the major imperfections of Algorithm 1 that arise due to partial volume effects. It is based on the observation that when a fixel is traversed by streamlines belonging to the pathway of interest in addition to other streamlines not belonging to that pathway, then rather than the entire volume of that fixel contributing to the pathway, ideally only the fraction of that fixel attributed to the pathway of interest should be included. This is achieved as follows (Figure 8; also pseudocode provided in Appendix 2):

1. Generate a whole-brain tractogram; define the pathway of interest as a subset of those whole-brain streamlines.

2. For every fixel, calculate the fraction of the total streamlines density in that fixel that belongs to the pathway of interest.

3. The contribution of the fibre volume of each fixel to the fibre volume of the pathway of interest is modulated by the fraction of that fixel ascribed to the pathway of interest in step 2.

4. As in Algorithm 1, divide the total volume of the pathway by the mean streamline length to estimate FBC.
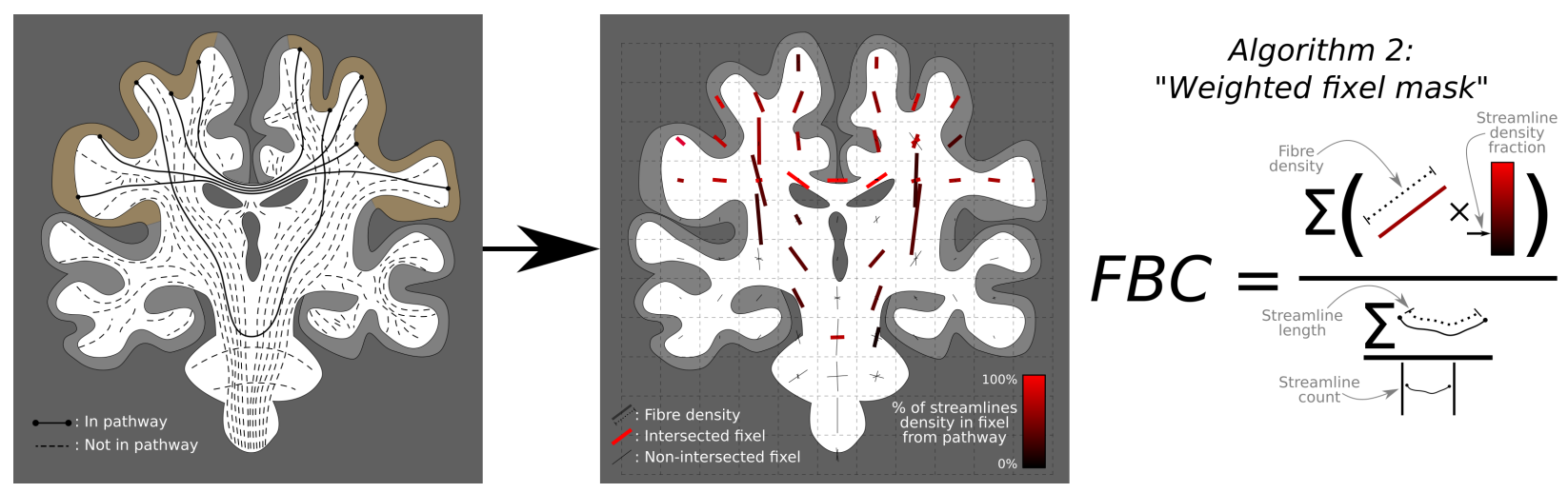

Figure 8: Visual demonstration of the operation of Algorithm 2 ("Weighted fixel mask"). The streamlines corresponding to the pathway of interest (left panel: solid lines) are a subset of a whole-brain tractogram (left panel: dashed lines). For each fixel in the image (right panel), the fraction of the streamlines density in that fixel corresponding to the pathway of interest can be quantified (right panel: red intensity). The contribution of the fibre volume within each fixel (encoded visually as fixel lengths) to the pathway of interest is modulated by the fraction of the streamlines density in that fixel attributed to the pathway of interest (multiplication in equation numerator); as with Algorithm 1, this volume is then divided by the mean streamline length (equation denominator: sum of streamline lengths divided by the number of streamlines) in the calculation of Fibre Bundle Capacity (FBC). 
The primary advantage of this approach over Algorithm 1 is that if a fixel is only traversed by a small number of streamlines within the pathway of interest, that fixel only contributes a small amount of its fibre volume to the FBC result. The effect of this change from Algorithm 1 is particularly evident in the inferior half of the brain in Figure 6b, where individual erroneous streamlines trajectories contribute far less to the calculated fibre pathway volume.

While various streamline reconstruction biases mean that the fixel fibre volume fractions ascribed to the streamlines within the pathway of interest may not be precisely equivalent to the total fraction of the underlying fibres within that fixel that belong to the biological pathway of interest, this algorithm certainly provides a perceptible improvement over Algorithm 1, with the spatial distribution of fibre volume within the pathway having a much more biologically plausible appearance.

An inherent problematic issue in the design of Algorithm 2 however is that it fails to enforce a consistent intra-axonal cross-sectional area within the pathway. Along the length of the fibre bundle shown in Figure 6b, there are local "hot-spots" of supposedly increased fibre volume, both within the superficial white matter and where the bundle intersects the grey matter targets. While individual axons may have some modulation in their diameter along their length, such gross modulation of macroscopic intra-axonal cross-sectional area is physically unrealistic for white matter pathways at the macro-scale. Observation of such in diffusion MRI data is therefore far more likely to be an artifact of image analysis and reconstruction. Furthermore, at the endpoints of the pathway, voxels containing partial volume between grey and white matter are likely to contain a smaller number of streamlines than those voxels entirely within the WM, which results in the fraction of fixel fibre density assigned to the pathway of interest being prone to quantisation effects; this contributes to the "speckly" appearance of the density map in Figure $6 \mathrm{~b}$ near the grey matter.

\section{Algorithm 3: "Volume-averaged streamline weights"}

In order to overcome the fundamental limitation of Algorithm 2, a solution is sought for deriving FBC that enforces a constant intra-axonal cross-sectional area along the length of the pathway. We clarify here that this constraint does not apply to the macroscopic span of space traversed by fibres of that bundle, but applies specifically to the intra-axonal portion of the bundle. For example: when fibres within a tightly-packed bundle diverge from one another (commonly referred to as "fanning"), the surface area of the subset of a plane encapsulating all fibres in the bundle may increase (the "macroscopic cross-section"), but the sum of intra-axonal cross-sectional areas should remain unchanged if the axon diameters are consistent along their length; here it is the latter definition that we advocate should be constrained.

Algorithm 3 is an initial realization of this concept. It enforces constant intra-axonal cross-sectional area of the pathway, by requiring that each streamline in the pathway contributes a constant fibre cross-sectional area along its entire length. Hence, unlike Algorithm 1 and Algorithm 2, here contributions toward FBC are made not per fixel, but per streamline (in the context of semi-global tractography algorithms, these parameters are sometimes referred to as "weights").

This algorithm operates as follows (Figure 9; also pseudocode provided in Appendix 3):

1. Using the whole-brain tractogram, calculate the total streamlines density in each fixel.

2. For each streamline, calculate the fibre volume to be attributed to that streamline. Every fixel traversed by the streamline contributes a fraction of its fibre volume to the sum for that streamline, based on the fraction of the total streamlines density in that fixel that was contributed by that particular streamline.

3. Convert the fibre volume of each streamline to a fibre cross-sectional area, by dividing by the length of that streamline.

4. Sum the cross-sectional areas of the streamlines belonging to the pathway of interest to derive FBC.

While this algorithm produces a measure of fibre cross-sectional area per streamline rather than fibre volume per fixel, it is still possible to reconstruct the latter; this allows us to generate a spatial map of fibre density attributed to the pathway of interest that can be compared to Algorithms 1 and 2. The product of a cross-sectional area with a length yields a measure of volume; hence, each streamline in the tractogram contributes a fibre volume to every voxel it traverses, based on the product of its weight and the length of the streamline segment that intersects that voxel. The result of this process is shown in Figure 6c. Compared to the previous two algorithms, this approach produces a fibre density map for the resulting pathway that appears quite biologically reasonable, with a maximal microscopic fibre density within the narrow confines of the corpus callosum that decreases as those fibres fan out toward the cortex. 


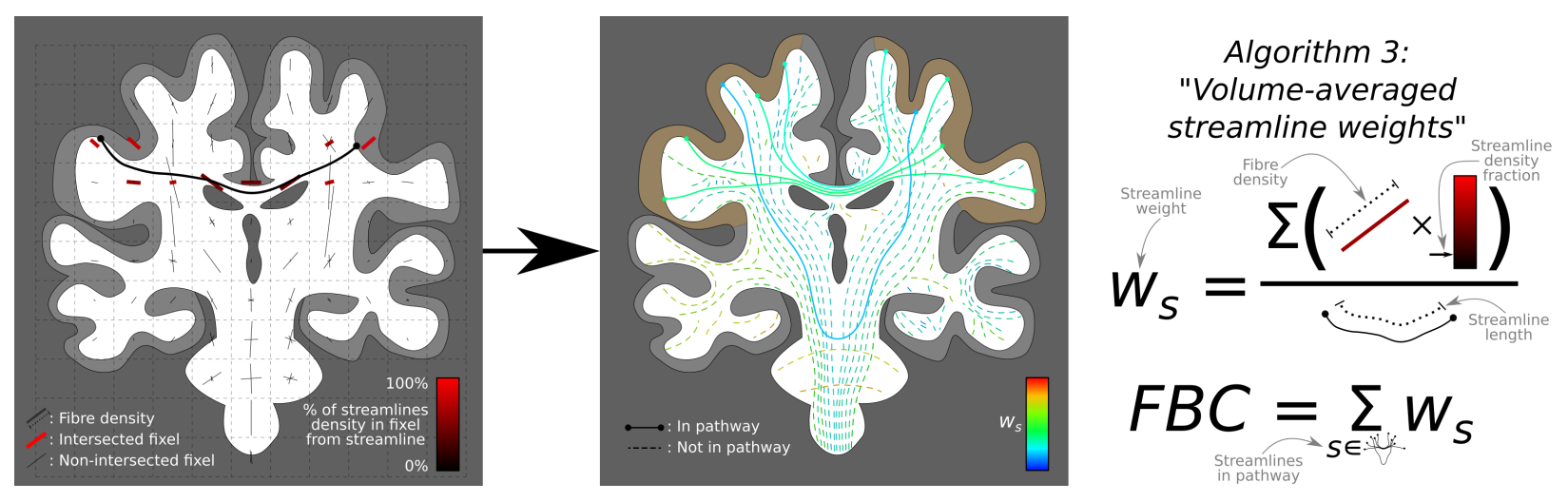

Figure 9: Visual demonstration of the operation of Algorithm 3 ("Volume-averaged streamline weights"). For each individual streamline, the total fibre volume attributed to that streamline (numerator, first equation) is based on the products of the fixel fibre volumes (encoded visually as fixel lengths) and the fraction of the streamlines density in each of those fixels attributed to the streamline of interest (left panel: encoded as red intensity); this fibre volume is then divided by the length of that individual streamline (denominator, first equation) to ascribe a "weight" $w_{s}$ to each individual streamline $s$ (Right panel: streamline colours). The Fibre Bundle Capacity (FBC) of the pathway of interest is then the sum of the weights ascribed to the streamlines attributed to the pathway of interest (right panel: solid lines).

There does however remain one slight inadequacy with this algorithm. Consider an experiment where, instead of deriving a spatial map of reconstructed fibre volume for a particular pathway of interest only (as we have been doing here), we instead map the spatial distribution of reconstructed fibre volume of the entire tractogram. If the streamlines weights are faithful to the intra-axonal cross-sectional areas of the biological fibres following the trajectories reconstructed by those streamlines, then one would expect an accurate reproduction of the fibre volumes that were estimated from the voxel-wise diffusion model throughout the white matter (or equivalently: applying the forward model to the whole-brain tractogram should yield the empirical diffusion signal, as demonstrated in the "Comparing tractograms and image data" section and Figure 3). Note this process is very similar to Track Density Imaging (TDI) at native DWI resolution[48-50], incorporating the ability for streamlines to contribute differentially toward the image.

This experiment is shown in Figure 10. The reconstructed fibre density from the outcome of Algorithm 3 (Figure 10c) is closer to the voxel-wise estimate of white matter fibre density derived from spherical deconvolution (Figure 10a $)$ than the original tractogram where every streamline contributes equally (Figure 10b). However, it still does not provide an entirely faithful representation of the underlying white matter fibre density field. This is perhaps expected given the nature of the algorithm itself. From a physical perspective, the operation of this algorithm can be thought of as taking the sum of fibre volumes attributed to the streamline by each fixel (which may be greater or lesser at different points along the streamline), and spreading this fibre volume evenly along the length of the streamline in order to ascribe to it a constant fibre cross-sectional area. While this process does go some way to incorporating fibre volume information from the diffusion model into the tractogram, it fails to directly enforce consistency between the estimated fibre density and reconstructed streamlines density for each fixel of the image (the residual discrepancy visible between Figure $10 \mathrm{a} \& \mathrm{c})$. In the case of the specific pathway of interest used in this demonstration, the fibre density within the corpus callosum projected by the tractogram is clearly greater than that indicated by the image data; this means that the calculated FBC for this connection relative to other pathways when using Algorithm 3 would likely be an over-estimate. 


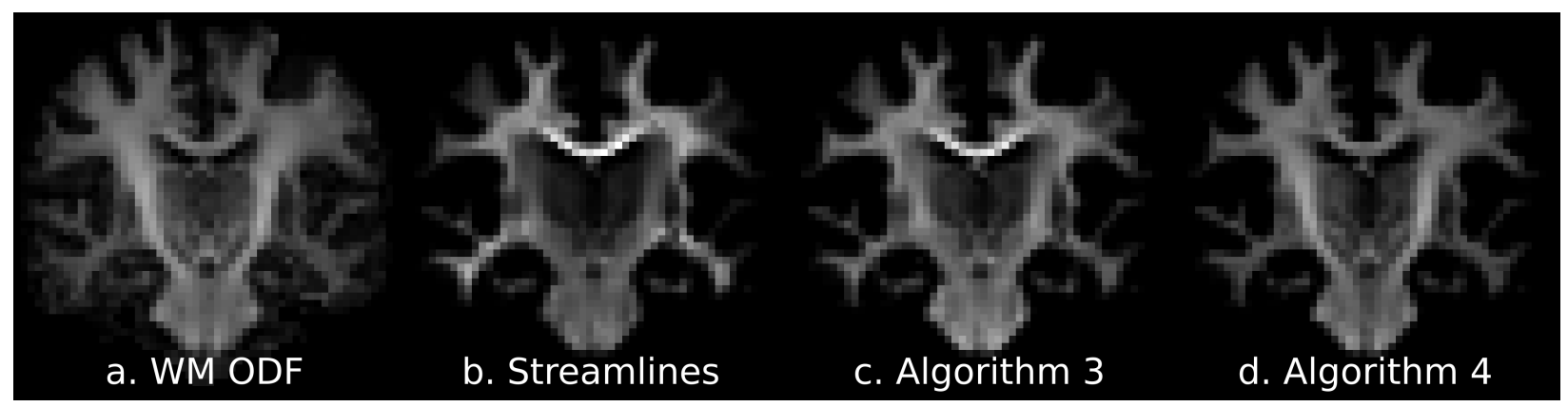

Figure 10: Comparison of spatial distributions of track densities from whole-brain tractogram data with the density of WM fibres as estimated through spherical deconvolution. a. The orientationally-averaged mean of the white matter Orientation Distribution Functions (the $l=0$ term of the spherical harmonic expansion) as a measure of total fibre density within each voxel. The distribution of track density within a whole-brain tractogram should ideally match these data. b-d. The density of streamlines in the whole-brain tractogram when the contribution of each streamline to the map is modulated as follows: $b$. No modulation (all streamlines contribute equally); c. modulated by the weights ascribed to the streamlines by Algorithm 3 ("Volume-averaged streamline weights"); d. modulated by the weights ascribed to the streamlines by Algorithm 4 ("Optimised streamline weights").

\section{Algorithm 4: "Optimized streamline weights"}

Addressing the remaining problem with the approach described in Algorithm 3 - the fact that the fibre volumes estimated from the tractogram are not a sufficiently accurate reconstruction of the fibre volumes estimated from the diffusion model, as shown in Figure 10 - is a fundamental requirement if we are to consider the streamline weights truly quantitative. If the streamlines trajectories and ascribed weights are reflective of the underlying biological connectivity, then the spatial distribution of fibre density throughout the white matter represented within this connectivity-based reconstruction should accurately match estimates of this measure that are derived from the image data directly.

This limitation is addressed by designing an algorithm that explicitly seeks to derive a set of streamline weights that result in an accurate reconstruction of the underlying fibre volumes estimated from the diffusion model (or equivalently, an accurate reconstruction of the empirical diffusion signal using a forward model). This basic concept is shown diagrammatically in Figure 11 (with pseudocode also provided in Appendix 4), and might proceed as e.g.:

1. Initially assign a unity weight to each streamline.

2. Using the whole-brain tractogram, based on the current streamline weights, calculate the total streamlines density in each fixel.

3. For each fixel, calculate the difference between the fibre density estimated from the diffusion model and the total attributed streamlines density.

4. For each streamline, increase or decrease the weight in order to minimise the error quantified in step 3.

5. Loop back to Step 2 until some termination criterion is met.

6. Sum the weights of those streamlines belonging to the pathway of interest to derive FBC.

This is the mechanism by which the "Spherical-deconvolution Informed Filtering of Tractograms 2 (SIFT2)" method[21] operates. The spatial distribution of fibre density within the pathway of interest after having applied the SIFT2 algorithm is shown in Figure 6d, and the spatial distribution of fibre density of the entire tractogram is shown in Figure 10d. Crucially, the latter demonstrates a highly accurate reproduction of the underlying fibre density field estimated

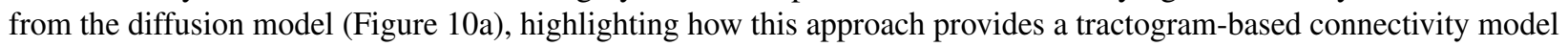
that obeys the fundamental spatial constraints imposed by the physical nature of the underlying biological fibre structure. It is this observation that permits the streamline weights estimated by such algorithms to be used in the quantification of FBC (within the constraints imposed by other limitations associated with diffusion MRI streamlines tractography[51]). 


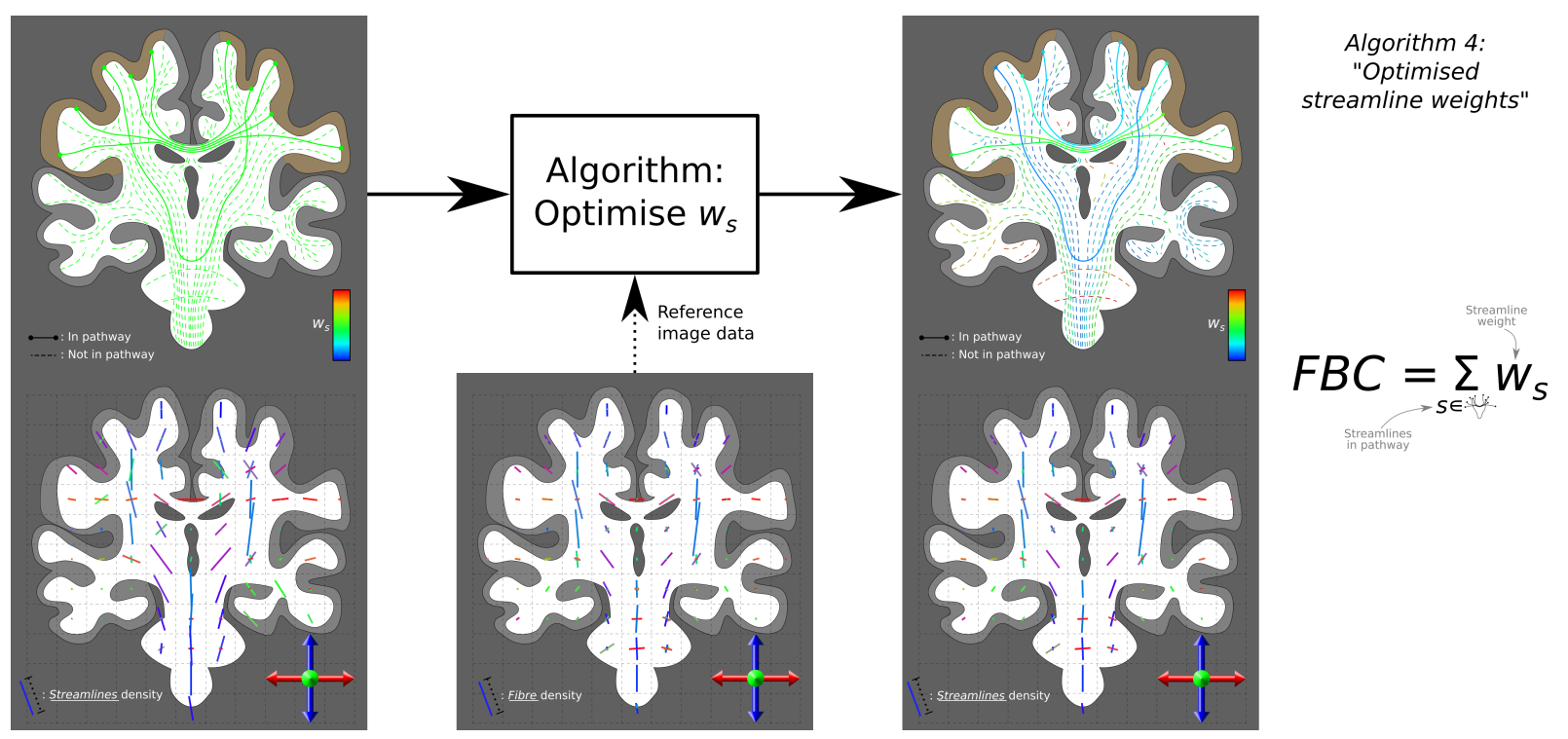

Figure 11: Visual demonstration of the operation of Algorithm 4 (“Optimised streamline weights"). For a whole-brain tractogram (top left panel), the total streamlines density traversing each fixel (bottom left panel; encoded visually as fixel lengths) may not match the fibre volumes estimated from the diffusion model (bottom panel; fixel lengths). This algorithm modulates the weight $w_{s}$ ascribed to each streamline $s$ (top right panel: streamline colours) in order to achieve correspondence between the total streamlines density traversing each fixel (bottom right; fixel lengths) and the diffusion model fibre density estimate (bottom panel). The Fibre Bundle Capacity (FBC) measure for the pathway of interest is the sum of the weights ascribed to those streamlines attributed to the pathway of interest (top right panel; solid lines only; streamline colours).

\section{Inter-subject connection density normalization}

Whenever quantitative data are to be compared across subjects, an important distinction must be made between absolute and relative quantitative measures. For instance, when assessing the Fractional Anisotropy (FA) measure, there is no need to modulate these values differentially between subjects, since it is an absolute measure. However, if a quantitative measure is relative to other latent parameters, care must be taken to properly regress out the effects of those latent parameters for the quantitative measure of interest to behave (as much as is possible) like an absolute measure.

In the Fixel-Based Analysis (FBA) framework[52], which enables statistical analysis of white matter quantitative measures in the presence of crossing fibres, fibre density estimates must be comparable across subjects throughout some common template space. In the context of Apparent Fibre Density (AFD) quantification using the spherical deconvolution model, FODs are deliberately not normalized either to a unit integral in each voxel, or to the intensity of the $b=0$ image in each voxel. This makes the size of the FOD directly proportional to the magnitude of the DWI signal (which is itself proportional to intra-cellular volume at high $b$-values[23]); the size of the FOD is also inversely proportional to the magnitude of the response function used for deconvolution[23]:

$$
\begin{gathered}
F O D * R F=D W I \\
F O D=D W I *^{-1} R F
\end{gathered}
$$

( $R F$ : response function; $*$ : convolution operation; $*^{-1}$ : deconvolution operation)

Enabling direct comparison of this measure across subjects therefore necessitates global inter-subject intensity normalization, in order for AFD to be minimally influenced by nuisance variables. This typically includes B1 bias field correction, scaling of DWI intensities to a common intensity value according to some representative image statistic (e.g. mean $b=0$ magnitude in WM), and use of a group average response function for deconvolution[23]: these together ensure that "one unit of AFD" is comparable across subjects, rather than being defined relative to subject-specific parameters (e.g. coil loading, scanner receiver gain, subject-specific response function magnitudes). 
In a similar manner, quantitative analysis of FBC requires inter-subject connection density normalization: that is, if we quantify the intra-axonal cross-sectional area of a particular pathway (e.g. edge of a connectome) across multiple subjects, we want these quantities to be directly comparable across subjects, without being biased by confounding factors that destroy the physical interpretation of this measure or introduce substantial correlations with nuisance reconstruction parameters.

In the brief history of diffusion MRI tractography connectomics, this normalization has most commonly been achieved by simply generating the same number of streamlines for each subject. Or, expressed in an alternative way: if half as many streamlines were generated for one subject as there were for all other subjects, it would seem intuitively logical that the streamline counts in each edge for that subject should be doubled in order for the raw values stored in the connectome matrices to be comparable to other subjects. While generation of an identical number of streamlines across subjects may seem incontrovertible, it is in fact an imperfect solution to connection density normalization. By using a fixed number of streamlines per subject, each streamline effectively represents a fixed, subject-specific fraction of the total white matter fibre connectivity. While comparing such quantitative measures across subjects is acceptable as long as they are properly interpreted as such, this metric fails to take into account a number of factors that may differ between subjects that may correspondingly introduce biases or unwanted variance into such an analysis; this includes both biological differences (e.g. widespread reductions in fibre density) and features of the tractogram reconstructions (e.g. differences in streamline lengths). For a genuine quantitative comparison of absolute fibre connection densities between subjects, greater care must therefore be taken.

Here we demonstrate our recommendation for how this normalization should be achieved when using specifically the SIFT model, which is itself directly dependent on the AFD measure. Use of alternative reconstruction techniques (either in terms of the underlying diffusion model, or alternative semi-global tractography methods) would require that appropriate comparable steps be taken.

The SIFT model defines the proportionality coefficient $\mu_{i}$ for subject $i$, which relates the global sum of track density $(T D)$ to the global sum of estimated fibre density $(F D)$ in the single-subject reconstruction, computed across all fixels $f$ in that subject:

$$
\mu_{i}=\frac{\sum_{f \in i} F D_{f}}{\sum_{f \in i} T D_{f}}
$$

(For simplicity, the influence of the processing mask within the SIFT model is omitted here)

In the original SIFT method, this parameter permits direct comparison between the streamlines density and fibre volume within each fixel, in order to drive the streamlines filtering process. In SIFT2, it approximately centres the distribution of streamlines weights about unity. Note that all parameters within this expression are subject-specific.

Since $F D$ is a measure of volume (dimensions $L^{3}$ ), and $T D$ is a sum of streamline lengths (dimensions $L$ ), $\mu$ is a measure of cross-sectional area, with dimensions $L^{2}$. For every streamline, this parameter (multiplied by the weight assigned to that streamline in the case of SIFT2) is a measure of the intra-axonal cross-sectional area represented by that streamline. For each voxel traversed by the streamline, the product of this cross-sectional area with the length of the streamline intersection within that voxel produces the fibre volume contributed to that fixel by that particular streamline within the model.

In the context of FBC quantification, we are interested not in these fixel-wise fibre volumes, but the connection densities of specific macroscopic pathways of interest. Any such pathway is represented as a subset of streamlines in the whole-brain tractogram. For an example pathway $p$ (which is reconstructed by a subset of streamlines $s$ ), it is the sum of intra-axonal cross-sectional areas of the streamlines within that pathway that gives a measure of the intra-axonal cross-sectional area of the pathway $F B C_{p, i}$ :

$$
F B C_{p, i}=\mu_{i} \cdot \sum_{s_{i} \in p} w_{s_{i}}
$$

For subject $i$, the connection density $F B C_{p, i}$ of pathway $p$ is the product of the subject-specific proportionality coefficient $\mu_{i}$ and the sum of streamline weights of those streamlines $s_{i}$ belonging to pathway $p$. 
(Note that in the original SIFT method, $w_{s}=1$ for all retained streamlines after filtering, but $\mu_{i}$ is modulated during the filtering process)

This equation suggests that if one wants to compare $F B C$ across subjects (whether for an individual bundle of interest or an entire connectome matrix), simply multiplying the sum of bundle streamlines weights by $\mu_{i}$ is sufficient to produce a measure of FBC that can be compared across subjects. However, parameter $\mu_{i}$ only considers the fibre densities and track densities within a single subject; in order to compare these quantities between subjects, we must ensure that parameter $\mu_{i}$ is adjusted appropriately to account for between-subject variation, by ensuring that the fundamental scaling underlying this parameter is equivalent between subjects.

We can extend Equation 3 as follows:

$$
\mu_{i, a d j}=\frac{A F D_{r e f}}{D W I_{r e f}} \cdot|x||y||z| \cdot \frac{\sum_{f \in i} F D_{f}}{\sum_{f \in i} T D_{f}}
$$

- $\mu_{i, a d j}$ is the proportionality coefficient of subject $i$ "adjusted" for facilitation of inter-subject comparison;

- The first term, $\frac{A F D_{\text {ref }}}{D W I_{r e f}}$, is specific to the spherical deconvolution model if processing were to be performed independently for each subject. It relates to the global scaling of AFD magnitudes within that subject, which is dependent on the magnitude of the diffusion-weighted signal $\left(D W I_{r e f}\right)$ and the size of the response function for deconvolution that forms the reference unit of $\operatorname{AFD}\left(A F D_{\text {ref }}\right)$.

- The second term, $|x||y||z|$, is the volume of each voxel in the image. This multiplier converts AFD (or fibre volume fractions from a partial volume-based diffusion model) into estimated intra-axonal volumes in $\mathrm{mm}^{3}$, thereby appropriately scaling connectivity estimates in cases where the DWI voxel size differs across subjects (this also coincidentally gives $\mu_{i, a d j}$, and hence FBC, units of $\mathrm{mm}^{2}$ ).

- In the specific case of the SIFT model, no term relating to the inter-subject scaling of track density appears in this expression: this is calculated in fixed units of $\mathrm{mm}$ regardless of DWI spatial resolution, and therefore cannot vary across subjects (this may however not be the case for alternative models or methods).

The global intensity normalisation and group average response function components of the recommended pre-processing pipeline for AFD analysis are tailored to make equivalent across subjects the values of $D W I_{\text {ref }}$ and $A F D_{\text {ref }}$ respectively. As such, if this pipeline is followed, term $\frac{A F D_{\text {ref }}}{D W I_{\text {ref }}}$ is identical across subjects by construction, and simply multiplying the sum of streamline weights within a pathway of interest (e.g. a connectome edge) by $\mu_{i}$ permits direct quantitative comparison of FBC between subjects, in a manner that appropriately accounts for many variables that would otherwise confound the interpretation of streamline counts as "connection density".

The consequences of this connection density normalisation are demonstrated in Figure 12. This demonstration consists of 16 individual synthetic subjects, each of whom possesses a single white matter fibre bundle. The fundamental properties of this bundle - length, width, and microscopic fibre density per voxel - vary among the subjects; additionally, the number of streamlines seeded in each white matter voxel is also varied. The lower part of Figure 12 then demonstrates visually how the quantified "connectivity" of this bundle across the 16 subjects changes in magnitude across the different subjects, depending on the exact measure of "connectivity" that is utilised. The connectivity measures demonstrated are:

- The streamline count $N$;

- The number of streamlines divided by the length of the streamlines[53];

- The mean fibre density $F D$ sampled along streamlines within the bundle;

- $F B C$, incorporating the proposed connection density normalisation.

We assert that the proposed connection density normalisation (highlighted in green in Figure 12) is most appropriate for quantitative comparison of endpoint-to-endpoint connectivity across subjects; it matches the theoretical properties of the FBC metric:

- Scales with the cross-sectional area of the bundle;

- Scales with the underlying fibre density in each voxel;

- Does not scale with the length of the bundle;

- Does not scale with the number of streamlines generated. 


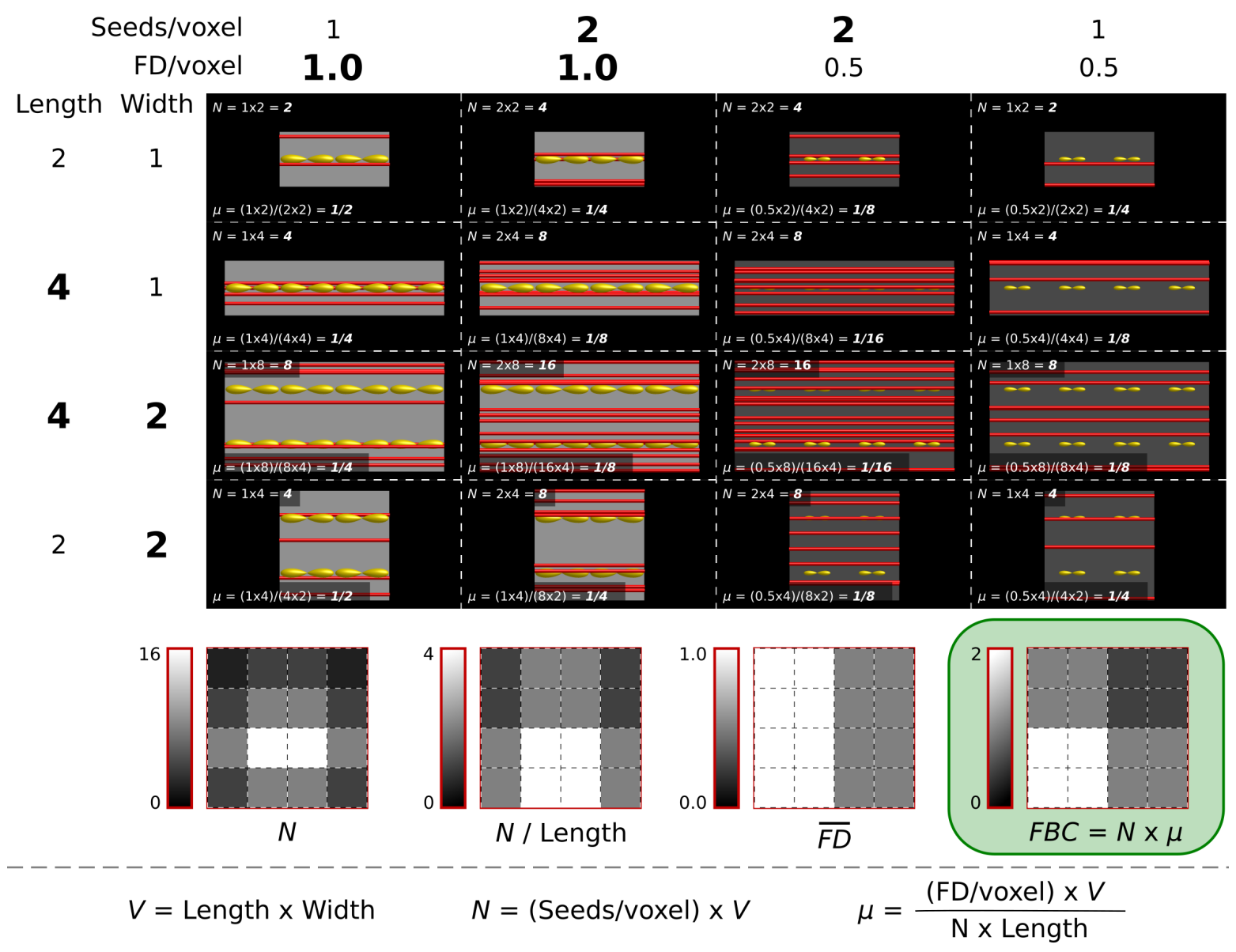

Figure 12: Demonstration of the efficacy of the proposed inter-subject connection density normalisation. Each of the sixteen panels represents a synthetic subject, containing one white matter bundle reconstructed by streamlines. For each, the number of streamlines generated $N$, and the "proportionality coefficient" $\mu$ within the SIFT model, are provided. The matrix representations at bottom show visually the relative connection densities quantified for the different bundles, for various "connectivity" metrics of interest: number of streamlines $N$; number of streamlines divided by the streamline length; the mean fibre density $F D$ sampled along streamlines within the bundle; the proposed FBC metric incorporating connection density normalisation (highlighted in green).

\section{Discussion}

As stated in the Introduction section, the information presented in the Methods section above is intended to serve two principal purposes:

1. To properly contextualise a class of methods already present in the literature that perform tractogram manipulation for the purposes of quantitative tractography by elucidating:

1. Why these methods are designed the way they are (see also "Relationship to existing methods" below);

2. That many "alternative" methods for white matter connectivity analysis frequently suggested informally by community members have already been considered, but have specific weaknesses compared to established methods;

3. That the goal of "quantifying bundle connectivity" is in fact accessible using these existing methods (while of course acknowledging all of the limitations of such methods and other components of the analysis pipeline).

2. To demonstrate how to appropriately extend the application of these methods from the correct estimation of relative connection strengths of different bundles within a single individual to estimation of relative connection strengths of a bundle across individuals (Figure 12). 


\section{Processing pipeline for FBC estimation}

Possibly the greatest misunderstanding in this context throughout the community is how these existing methods relate to the quantification of connectivity of individual bundles of interest. It is not immediately obvious why, when attempting to quantify the density of a specific bundle of interest, a method that explicitly operates on whole-brain tractograms is even applicable. But what is shown in the contrast between Algorithm 1 and the other algorithms is that, if structural connections outside of the pathway of interest are not considered, such quantification will be corrupted anywhere that there is any form of spatial overlap of pathways (e.g. Figure $6 \& \underline{\text { Figure 7) }}$.

The appropriate sequence of processing steps to quantify FBC for a specific pathway of interest (regardless of whether there is only one such pathway, or one is interrogating all possible pathways to generate the brain structural connectome), is (see also Figure 11):

1. Generate a whole-brain tractogram;

2. Apply a semi-global tractogram optimisation method that assigns weights to individual streamlines;

3. Select a subset of streamlines from the whole-brain tractogram that corresponds to the pathway of interest;

4. FBC is the sum of the streamline weights calculated in step 2 ascribed to the streamlines selected in step 3.

Any deviation from this process_-for instance, the discarding of specific streamlines in between steps 1 and 2[54,55] may detrimentally influence the quantification of FBC, by effectively re-introducing those weakness exhibited by Algorithm 1.

\section{Relationship to existing methods}

In "Algorithm 4: "Optimized streamline weights"”, the fact that the existing SIFT2 algorithm operates on the same mechanism as that arrived at through the course of logic presented is not coincidental. Here we note that there are a number of other existing methods that also operate similarly:

- The "Linear Fascicle Evaluation (LiFE)" [20] and "Convex Optimisation Modeling for Microstructure Informed Tractography (COMMIT)"[19] methods both operate on an identical premise - modulating the weight of contributions from individual streamlines within the tractogram, in such a way that the streamlines densities are faithful to the underlying image information - except that they operate directly on the diffusion image data, as explained in the "Comparing tractograms and image data" section.

- The earlier "BlueMatter"[17], "MicroTrack"[56] and "Spherical-deconvolution Informed Filtering of Tractograms (SIFT)"[18] methods also lie within this classification: while these algorithms instead select a subset of streamlines that together produce a faithful representation of the image data, this is mathematically equivalent to setting the contribution weights of those streamlines omitted from the tractogram to zero.

- Another method entitled "global tractography with embedded anatomical priors"[57] optimises streamline weights based on a tractogram initially constructed using streamlines tractography, but also optimises other features of the reconstruction (such as streamlines trajectories) based on the diffusion image data in a manner more similar to genuinely global tractography algorithms.

\section{Alternatives for connection density normalization}

A common idea in applications of white matter tractography, particularly in the construction of the structural connectome, is that there is a range of other parameters that should be applied as multiplicative factors to connectivity measures, in order to "compensate" for variations in those parameters that may indirectly influence the results of streamlines tractography or connectome construction. Here we take the opportunity to clarify a few concepts that have arisen in our own communications on the topic.

\section{Number of streamlines in the connectome}

Although it is trivial to use a fixed number of streamlines for tractogram construction across subjects for the implicit purpose of connection density normalisation, typically the proportion of those streamlines successfully assigned to a pair of parcels (and that hence contribute to the connectome) will vary between subjects[58], due to a range of factors; 
note that this still occurs even with the controlled termination of streamlines such as in Anatomically-Constrained Tractography (ACT)[15], though technical improvements can reduce this variance[59,60]. While it is tempting to normalise the connectome edge values by scaling for the number of streamlines in the connectome (rather than using the number of streamlines in the tractogram as reference) to "compensate" for this inter-subject discrepancy, it is also entirely possible for differences in this parameter to be reflective of a genuine effect of interest.

For instance, consider the case where a large tumor within the white matter in one hemisphere results in a substantial number of streamlines terminating within that tumor, rather than reaching some alternative grey matter target. If the subject-specific tumor does not appear as a node within the connectome parcellation, and the total number of streamlines generated across subjects is equivalent, then the total number of streamlines in the connectome for this particular subject would be decreased relative to other subjects (since the total number of streamlines is the same, but the fraction of those assigned to the connectome is reduced). A useful interpretation here is to treat the tumor as a "latent connectome node". Consider the situation if the tumor were to be segmented and included in the connectome parcellation, with streamlines terminating within that node and being assigned as such; but following connectome construction, that node would then be erased from the connectome matrix. We now consider two options for normalisation:

1. If a fixed number of streamlines in the tractogram per subject were to be used, then the subject with the tumor would have a reduced total connection density within the connectome, particularly within bundles affected by the tumor, which is likely to be at least somewhat faithful to biological reality.

2. If instead the connectomes were scaled based on the number of streamlines in the connectome in each subject (bearing in mind that this scaling would by necessity occur after removal of the tumor node if the total connection density is to be equivalent across subjects), the connection densities of all pathways in that subject would be increased as a consequence of that process. This would be misleading, as it would suggest that all white matter bundles not affected by the tumor in that subject have increased connectivity in that subject relative to healthy controls.

The important observation here is that while the number of streamlines in the tractogram reconstruction may not be equal to the number of streamlines in the connectome (and this ratio may vary across participants), and this effect can be influenced by inadequacies in data processing and reconstruction[58], this is not the only source of such mismatch, and should therefore be interpreted with caution. For instance, consider the influence of the reconstructed cortico-spinal tract, where streamlines exit the inferior edge of the image data via the spinal column. If no connectome parcel is explicitly defined at this location, then these streamlines will not contribute to the connectome, despite the known anatomical validity of this bundle and its non-negligible contribution to the diffusion-weighted signal. Variance in the density of this bundle across subjects could manifest as differences in streamline count within connectomes across subjects; the latter would therefore not ideally be interpreted as either indicative of a difference in reconstruction efficacy, or a "nuisance" effect between individuals,

\section{Intracranial / brain / white matter volume}

Another concept commonly raised in discussion on this topic is that: if subject brains vary considerably in physical size, but the same number of streamlines is generated for each, then a comparison of streamlines density between them must be biased, since each individual streamline reconstructed in a physically larger brain likely represents a larger density of biological fibres than does each individual streamline reconstructed in a physically smaller brain. Users of such methods correspondingly often propose dividing all estimates of connectivity by the estimated intracranial / brain / white matter volume of that subject, as a "correction" for this effect. There are a number of comments to be made on this concept:

- It pre-supposes that if the cross-sectional area of a bundle scales directly in proportion to brain size, then that bundle should be reported as possessing equivalent "connectivity" between brains of different size; this is the intent of such scaling, and so should be appreciated as such.

- There is an implicit assumption in this proposal that, even between the largest and smallest of brains, the voxel-wise fibre density within the white matter is equivalent; this is however not guaranteed to be the case.

- Performing scaling as proposed in the "Inter-subject connection density normalisation" section intrinsically handles this confound in an appropriate fashion. A larger brain will likely have more white matter voxels and therefore a larger fibre density sum, but it will also have a greater sum of streamline lengths. Consequently, while FBC may be greater in a large brain than in a small brain if the bundle size scales in direct proportion to brain size, this would not be an unwanted confound of brain size, but a realistic measurement of a greater 
information-carrying capacity of that fibre bundle in the larger brain.

- The interpretation of experimental outcomes also changes by necessity through such scaling. For instance, if one were to compare the connection density of a specific bundle of interest between two groups, where this brain volume scaling factor were applied to the connectivity estimates prior to the comparison, then the actual hypothesis being tested would be "the connection-density-divided-by-white-matter-volume of this pathway is not equivalent between two groups".

We instead suggest that intracranial / brain volume (and other such parameters) may be better handled as nuisance parameters when performing statistical testing. In this way, the hypothesis being tested would better reflect the intention of the experiment, e.g. "the connection density of this pathway is not equivalent between the two groups, the magnitude of which cannot be attributed to differences in brain volume alone".

\section{The effect of inter-node distance in streamlines tractography}

The relationship between white matter pathway length and estimated connection density has attracted considerable interest in this field[18,53,61]. There are however multiple mechanisms by which pathway length may influence streamlines-based connectivity; and in our experience these are regularly conflated or confused. We therefore take this opportunity to disambiguate the effects of which we are aware.

Most frequently, discussion regarding bundle length biases are in reference to the effect arising from homogeneous seeding throughout the white matter: because longer pathways present a greater volume in which streamline seeds may be drawn, they will typically be reconstructed by a greater number of streamlines than shorter pathways. A naïve direct correction of this seeding density effect is to make the contribution of each streamline to the connectome the reciprocal of its length[53]; this has been shown to be incomplete, as the graph theory metrics derived from connectomes calculated in such a manner differ significantly from those produced using more comprehensive data-driven correction of fibre tracking biases[61].

A distinctly different effect is attributed to probabilistic streamlines algorithms. Due to the spatial dispersion of streamlines when using a probabilistic tracking algorithm, biologically-connected nodes that are distant from one another are likely to have a reduced reconstructed connection density: streamlines emanating from one parcel increasingly disperse from one another as a function of distance from that parcel, such that the fraction of those streamlines reaching the intended target decreases as a function of distance ${ }^{1}$. This effect is however not a bias that can be corrected naively. For instance, consider two distant nodes that are $\overline{n o t}$ connected biologically, yet their immediate spatial neighbouring parcels are connected biologically, and therefore there is a plausible white matter pathway between them. The connectivity estimated between these two nodes using a probabilistic streamlines algorithm will be increased by this probabilistic dispersion effect relative to if the white matter pathway were short. Data regarding the inter-node distance alone is therefore not sufficient to "correct" for this effect.

We propose that this particular effect is therefore better understood as a distance-dependent connectome blurring: biologically strong connections are "spread out" in the reconstruction to edges corresponding to spatially adjacent nodes, with the extent of that blurring being a function of the pathway length. The way in which fibre orientation uncertainty / dispersion is modelled and utilized in the tractography algorithm also is likely to influence the magnitude of this effect. While there exists a tailored correction mechanism for addressing this specific issue in the context of targeted tracking when quantifying a probability of connectivity[62], to our knowledge there has been no such mechanism proposed for addressing this issue when quantifying the density of white matter connections.

We further clarify that there is another streamlines tractography effect that bears similarity to that described above, but behaves slightly differently, and applies to both deterministic and probabilistic streamlines algorithms. Opportunities for the streamlines algorithm to sample from an inappropriate fibre orientation (particularly in the presence of crossing fibres), and therefore construct a wholly erroneous trajectory, increase as a function of bundle length. This effect has also to our knowledge not been investigated comprehensively but should be considered as distinct from both other "influences of bundle length on streamline count" described above.

\footnotetext{
${ }^{1}$ We note that deterministic streamlines algorithms do not solve the issue described here. With such methods, instead of the fraction of streamlines reaching the intended target decreasing smoothly as a function of distance, the likelihood of a dichotomous switch from all true connections to all false connections increases as a function of distance.
} 


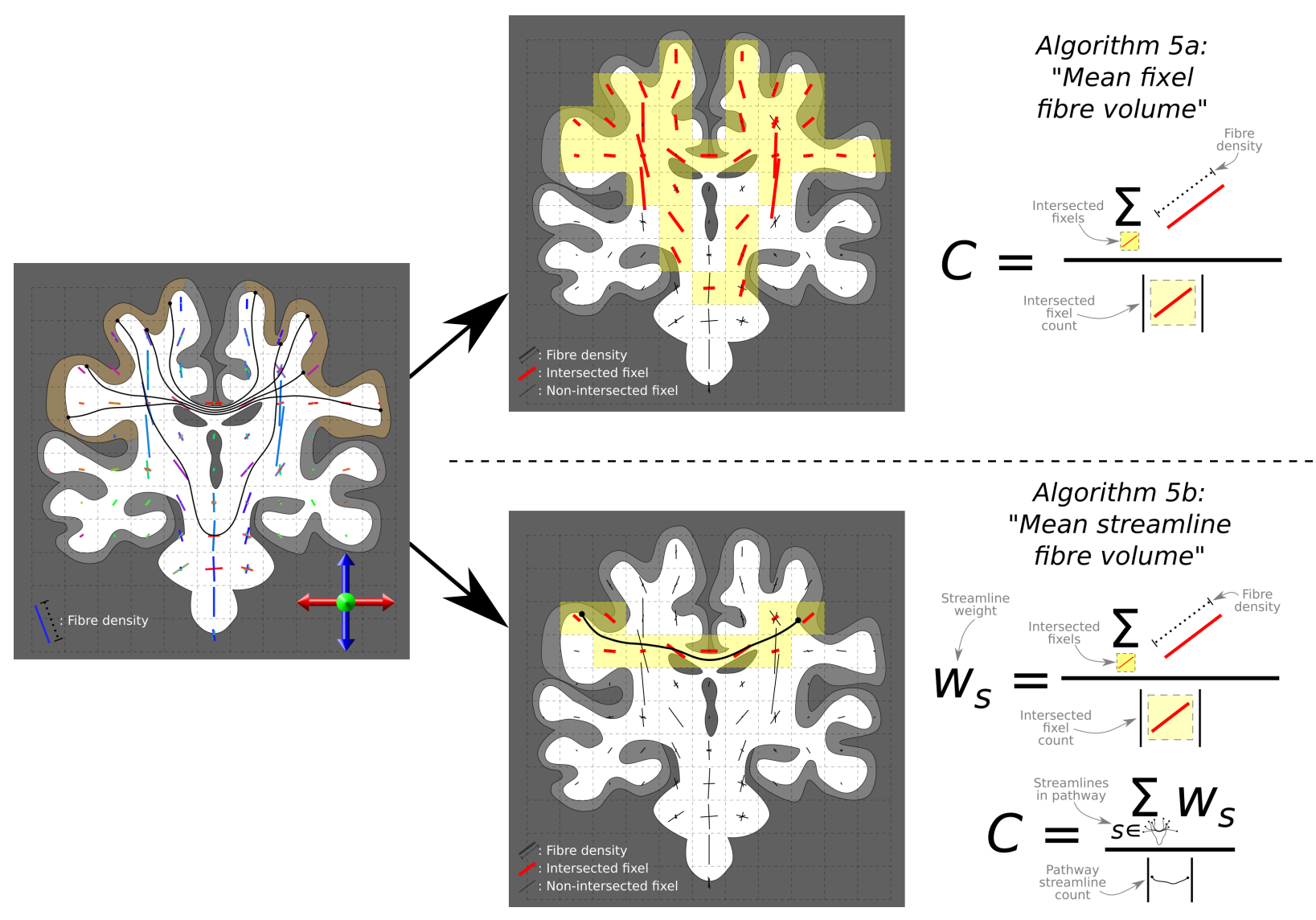

Figure 13: Visual demonstration of the operation of hypothetical alternative Algorithms 5a and 5b. a. For the pathway of interest (left panel; streamlines), derive a mask of fixels traversed (top panel; red fixels within yellow voxels). Within this mask, compute from the fibre densities of those fixels (encoded visually as fixel lengths) the mean (equation at right shows the sum of fixel fibre densities divided by the number of traversed fixels), as a measure of "connectivity" $C$ of the pathway. b. For each individual streamline within the pathway of interest (left panel), derive a mask of fixels traversed by that streamline (bottom panel; red fixels). Across those fixels, compute the mean fibre density (first equation at right shows the sum of traversed fixel fibre densities divided by the number of fixels traversed by that streamline). Finally, take the mean of these values across the streamlines corresponding to the pathway of interest (second equation at right shows sum of streamline weights divided by the number of streamlines) as a measure of "connectivity" $C$ of the pathway.

\section{Relationship to fixel-based analysis metrics}

We have recently published on the disentanglement of statistical effects in microscopic fibre density and macroscopic changes in cross-sectional area, made possible within the Fixel-Based Analysis (FBA) framework[52]. Due to the subsequent interest we received in the potential incorporation of such fixel-wise measures into tractogram and/or structural connectome quantification, here we clarify the relationship between these fixel-wise measures and FBC.

For any particular white matter pathway in the brain, FBC quantified using a global or semi-global tractography approach will scale directly proportionally with both the microscopic fibre density (resulting in a greater number of streamlines or increased streamlines weights traversing any particular fixel) and the macroscopic pathway cross-sectional area (resulting in a greater breadth of fixels traversed by the streamlines within that pathway, and hence likely also more streamlines being assigned to that pathway), as shown in Figure 12. FBC quantified in this manner therefore behaves most comparably to the "Fibre Density and bundle Cross-section (FDC)" measure[52]; but crucially, FBC is quantified as an endpoint-to-endpoint connectivity measure, whereas FDC is a local fixel-wise quantitative measure (as shown in Figure 2).

There is an additional clarification required regarding terminology between these two cases: 
- In FBA, the "Fibre bundle Cross-section (FC)" metric is in fact a change in cross-sectional area; in calculation of the FDC metric, this is used to modulate the local quantitative measure;

- In tractogram-based connectivity quantification, FBC of any particular pathway is directly proportional to the absolute bundle cross-sectional area.

Another concept frequently raised in communications in this context is the prospect of an alternative quantitative metric for connectome construction, which exploits the quantitative nature of these per-fixel metrics. That is, instead of summing streamlines weights within a pathway (as an estimate of intra-axonal cross-sectional area), quantitative values from some metric of interest are instead sampled along the corresponding streamlines trajectories. This could conceivably be done in one of two ways, shown as Algorithms 5a and 5b in Figure 13 (with pseudocode also provided in Appendix 5a and Appendix 5b respectively):

- From the set of streamlines constituting a pathway of interest / connectome edge, derive a mask corresponding to the areas (either voxels or fixels) in which the values of the quantitative metric should be sampled; some statistic from these elements (e.g. the mean) is then calculated to produce a single scalar value per connectome edge.

- For each streamline, measure the value of the metric at every point along the streamline trajectory; calculate some statistic from the samples along each streamline (e.g. the mean) in order to produce a single scalar value per streamline; calculate some statistic from these per-streamline values (e.g. the mean) to produce a single scalar value per connectome edge.

The intent behind such suggestions is that these quantities would exhibit a reduced influence from the errors and biases associated with streamlines tractography compared to the FBC metric, would incorporate the quantitative nature of those underlying metrics, and would inherit the fixel specificity of the FBA metrics (there are already many applications that have utilised such methods but in conjunction with voxel-wise imaging metrics). Such quantification should however be interpreted in accordance with the relevant calculations. For instance, calculating the mean of the Fibre Density (FD) metric along a pathway using one of the two approaches above provides a measure that could be interpreted as "mean intra-axonal volume fraction within the bundle"; which, while potentially informative, would not be an absolute measure of bundle connectivity, as it neither scales with bundle width (Figure 12), nor does it consider partial volume with other bundles (as demonstrated in Algorithm 1, Figure 6 and Figure 7).

\section{Software implementations}

The algorithms described in the section "The algorithmic basis for quantitative white matter tractography" have been made available as part of the MRtrix3 software package[63] (www.mrtrix.org; command and option names as at version 3.0 .0$)$ :

- Algorithm 1, "Fixel mask": afdconnectivity;

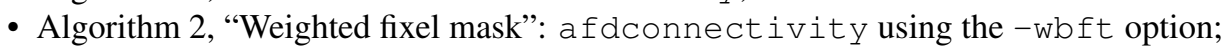

- $\overline{\text { Algorithm } 3}$, "Volume-averaged streamline weights": tcksift 2 using the -linear option;

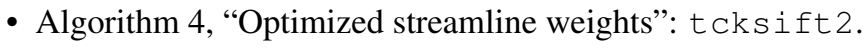

The SIFT method[18] mentioned in the "Relationship to existing methods" section is additionally available as command tcksift.

\section{Conclusion}

We have shown how the algorithmic design of a class of "semi-global" tractogram optimisation algorithms is the inevitable result of trying to incorporate global image information into otherwise locally-greedy, streamlines-based tractography data. When used appropriately, these methods address one of the major fundamental technical limitations in the field that otherwise precludes the direct comparison of quantitative estimates of white matter connection density between subjects. We hope that the explanations and clarifications contained herein assist those readers for whom the purpose (or indeed existence) of these approaches was unclear. 


\section{Acknowledgments}

We are grateful to the National Health and Medical Research Council (NHMRC) of Australia, and the Victorian Government's Operational Infrastructure Support Program for their support.

RS is supported by fellowship funding from the National Imaging Facility (NIF), an Australian Government National Collaborative Research Infrastructure Strategy (NCRIS) capability.

JDT was supported with funding from the European Research Council under the European Union's Seventh Framework Programme [FP7/20072013], ERC grant agreement no. [319456] (developing Human Connectome Project) and MRC strategic funds [MR/K006355/1]. JDT was also supported by the Wellcome/EPSRC Centre for Medical Engineering at King's College London [WT 203148/Z/16/Z] and by the National Institute for Health Research (NIHR) Biomedical Research Centre at Guy's and St Thomas' NHS Foundation Trust and King's College London. The views expressed are those of the authors and not necessarily those of the NHS, the NIHR or the Department of Health.

\section{Appendix: Pseudocode}

The code snippets provided below are intended only to demonstrate the fundamental logic behind the algorithms presented in code for. While the Python language is used as the basis for pseudocode syntax, none of the code is not intended to be executable.
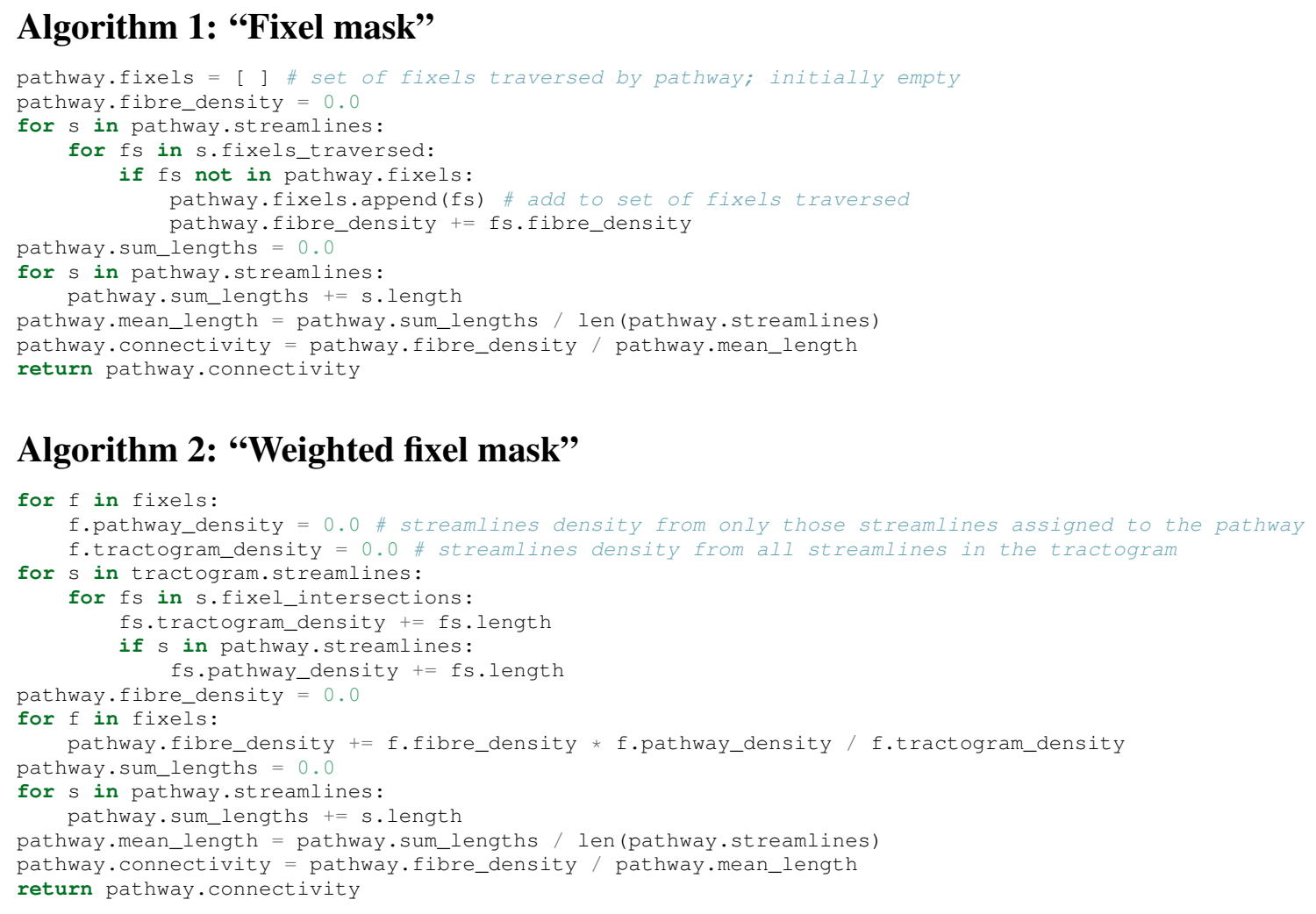

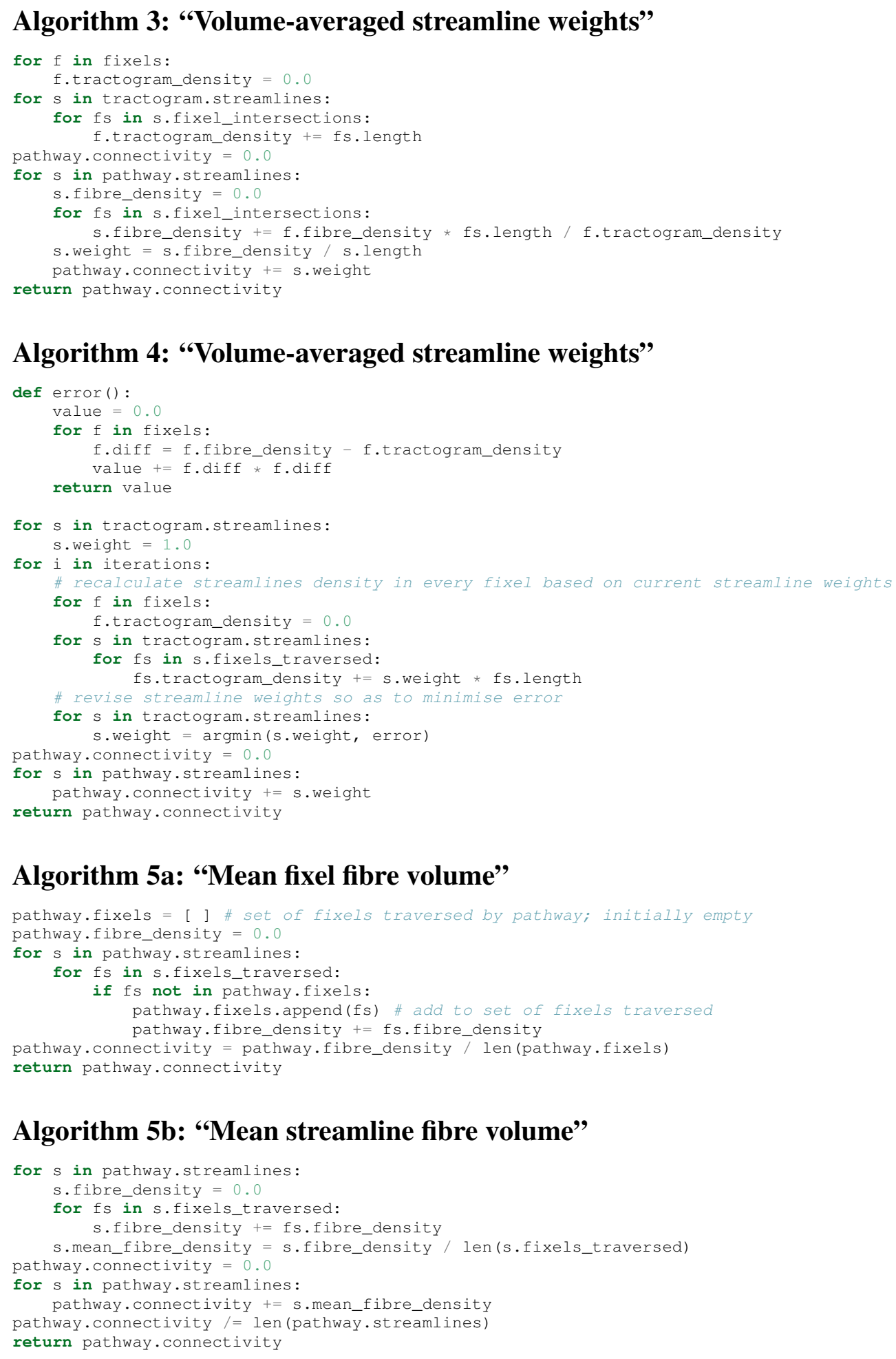


\section{References}

[1] Mori S, Zijl PCM van. Fiber tracking: Principles and strategies - a technical review. NMR in Biomedicine 2002;15:468-80.

[2] Dell'acqua F, Catani M. Structural human brain networks: Hot topics in diffusion tractography. Current Opinions in Neurology 2012;25:375-83.

[3] Johansen-Berg H, Behrens TEJ. Just pretty pictures? What diffusion tractography can add in clinical neuroscience. Current Opinion in Neurology 2006;19:379-85.

[4] Griffa A, Baumann PS, Thiran J-P, Hagmann P. Structural connectomics in brain diseases. Mapping the Connectome 2013;80:515-26.

[5] Mori S, Crain BJ, Chacko VP, Zijl PCM van. Three-dimensional tracking of axonal projections in the brain by magnetic resonance imaging. Annals of Neurology 1999;45:265-9. https://doi.org/10.1002/1531-8249(199902)45: 2\%3C265::aid-ana21\%3E3.0.co;2-3.

[6] Conturo TE, Lori NF, Cull TS, Akbudak E, Snyder AZ, Shimony JS, et al. Tracking neuronal fiber pathways in the living human brain. Proceedings of the National Academy of Sciences of the United States of America 1999;96:10422-7.

[7] Basser PJ, Pajevic S, Pierpaoli C, Duda J, Aldroubi A. In vivo fiber tractography using DT-MRI data. Magnetic Resonance in Medicine 2000;44:625-32.

[8] Jeurissen B, Descoteaux M, Mori S, Leemans A. Diffusion MRI fiber tractography of the brain. NMR in Biomedicine 2019;32:e3785. https://doi.org/10.1002/nbm.3785.

[9] Jones DK, Knösche TR, Turner R. White matter integrity, fiber count, and other fallacies: The do's and don'ts of diffusion MRI. NeuroImage 2013;73:239-54.

[10] Mangin J-F, Poupon C, Cointepas Y, Rivière D, Papadopoulos-Orfanos D, Clark CA, et al. A framework based on spin glass models for the inference of anatomical connectivity from diffusion-weighted MR data - a technical review. NMR in Biomedicine 2002;15:481-92.

[11] Kreher BW, Mader I, Kiselev VG. Gibbs tracking: A novel approach for the reconstruction of neuronal pathways. Magnetic Resonance in Medicine 2008;60:953-63.

[12] Reisert M, Mader I, Anastasopoulos C, Weigel M, Schnell S, Kiselev V. Global fiber reconstruction becomes practical. NeuroImage 2011;54:955-62.

[13] Mangin J-F, Fillard P, Cointepas Y, Le Bihan D, Frouin V, Poupon C. Toward global tractography. Mapping the Connectome 2013;80:290-6.

[14] Christiaens D, Reisert M, Dhollander T, Sunaert S, Suetens P, Maes F. Global tractography of multi-shell diffusionweighted imaging data using a multi-tissue model. NeuroImage 2015;123:89-101.

[15] Smith RE, Tournier J-D, Calamante F, Connelly A. Anatomically-constrained tractography: Improved diffusion MRI streamlines tractography through effective use of anatomical information. NeuroImage 2012;62:1924-38.

[16] Girard G, Descoteaux M. Anatomical Tissue Probability Priors for Tractography. In:. CDMRI, 2012, pp. 174-85.

[17] Sherbondy AJ, Dougherty RF, Ananthanarayanan R, Modha DS, Wandell BA. Think Global, Act Local; Projectome Estimation with BlueMatter. In:. Medical Image Computing and Computer-Assisted Intervention, London, UK: Springer-Verlag; 2009, pp. 861-8.

[18] Smith RE, Tournier J-D, Calamante F, Connelly A. SIFT: Spherical-deconvolution informed filtering of tractograms. NeuroImage 2013;67:298-312.

[19] Daducci A, Dal Palú A, Lemkaddem A, Thiran J. COMMIT: Convex Optimization Modeling for Micro-structure Informed Tractography. In:. Medical Imaging, IEEE Transactions on, vol. 34, 2014, pp. 246-57. https://doi.org/10. 1109/TMI.2014.2352414.

[20] Pestilli F, Yeatman JD, Rokem A, Kay KN, Wandell BA. Evaluation and statistical inference for human connectomes. Nat Meth 2014;11:1058-63. 
[21] Smith RE, Tournier J-D, Calamante F, Connelly A. SIFT2: Enabling dense quantitative assessment of brain white matter connectivity using streamlines tractography. NeuroImage 2015;119:338-51.

[22] Smith RE, Tournier J-D, Calamante F, Connelly A. The effects of SIFT on the reproducibility and biological accuracy of the structural connectome. NeuroImage 2015;104:253-65.

[23] Raffelt D, Tournier J-D, Rose S, Ridgway GR, Henderson R, Crozier S, et al. Apparent Fibre Density: A novel measure for the analysis of diffusion-weighted magnetic resonance images. NeuroImage 2012;59:3976-94.

[24] Drobnjak I, Zhang H, Ianuş A, Kaden E, Alexander DC. PGSE, OGSE, and sensitivity to axon diameter in diffusion MRI: Insight from a simulation study. Magnetic Resonance in Medicine 2016;75:688-700. https://doi.org/10.1002/ $\underline{\operatorname{mrm} .25631}$.

[25] Whittall KP, Mackay AL, Graeb DA, Nugent RA, Li DKB, Paty DW. In vivo measurement of T2 distributions and water contents in normal human brain. Magnetic Resonance in Medicine 1997;37:34-43. https://doi.org/10.1002/mrm. 1910370107.

[26] Swadlow H, Waxman S. Axonal conduction delays. Scholarpedia 2012;7:1451.

[27] Savadjiev P, Campbell JSW, Descoteaux M, Deriche R, Pike GB, Siddiqi K. Labeling of ambiguous subvoxel fibre bundle configurations in high angular resolution diffusion MRI. NeuroImage 2008;41:58-68.

[28] Close TG, Tournier J-D, Johnston LA, Calamante F, Mareels I, Connelly A. Fourier Tract Sampling (FouTS): A framework for improved inference of white matter tracts from diffusion MRI by explicitly modelling tract volume. NeuroImage 2015;120:412-27.

[29] Daducci A, Dal Palú A, Descoteaux M, Thiran J-P. Microstructure Informed Tractography: Pitfalls and Open Challenges. Frontiers in Neuroscience 2016;10:247.

[30] Girard G, Daducci A, Petit L, Thiran J-P, Whittingstall K, Deriche R, et al. AxTract: Toward microstructure informed tractography. Hum Brain Mapp 2017;38:5485-500.

[31] Raffelt DA, Smith RE, Ridgway GR, Tournier J-D, Vaughan DN, Rose S, et al. Connectivity-based fixel enhancement: Whole-brain statistical analysis of diffusion MRI measures in the presence of crossing fibres. NeuroImage 2015;117:40-55.

[32] Behrens TEJ, Woolrich MW, Jenkinson M, Johansen-Berg H, Nunes RG, Clare S, et al. Characterization and propagation of uncertainty in diffusion-weighted MR imaging. Magnetic Resonance in Medicine 2003;50:1077-88.

[33] Dell'Acqua F, Rizzo G, Scifo P, Clarke RA, Scotti G, Fazio F. A Model-Based Deconvolution Approach to Solve Fiber Crossing in Diffusion-Weighted MR Imaging. Biomedical Engineering, IEEE Transactions on 2007;54:462-72. https://doi.org/10.1109/TBME.2006.888830.

[34] Alexander DC, Hubbard PL, Hall MG, Moore EA, Ptito M, Parker GJM, et al. Orientationally invariant indices of axon diameter and density from diffusion MRI. NeuroImage 2010;52:1374-89.

[35] Assaf Y, Basser PJ. Composite hindered and restricted model of diffusion (CHARMED) MR imaging of the human brain. NeuroImage 2005;27:48-58.

[36] Assaf Y, Blumenfeld-Katzir T, Yovel Y, Basser PJ. AxCaliber: A method for measuring axon diameter distribution from diffusion MRI. Magnetic Resonance in Medicine 2008;59:1347-54.

[37] Behrens TEJ, Johansen-Berg H, Jbabdi S, Rushworth MFS, Woolrich MW. Probabilistic diffusion tractography with multiple fibre orientations: What can we gain? NeuroImage 2007;34:144-55.

[38] Kaden E, Kelm ND, Carson RP, Does MD, Alexander DC. Multi-compartment microscopic diffusion imaging. NeuroImage 2016;139:346-59.

[39] Panagiotaki E, Schneider T, Siow B, Hall MG, Lythgoe MF, Alexander DC. Compartment models of the diffusion MR signal in brain white matter: A taxonomy and comparison. NeuroImage 2012;59:2241-54.

[40] Zhang H, Schneider T, Wheeler-Kingshott CA, Alexander DC. NODDI: Practical lem in vivo neurite orientation dispersion and density imaging of the human brain. NeuroImage 2012;61:1000-16. 
[41] Tournier J-D, Calamante F, Gadian DG, Connelly A. Direct estimation of the fiber orientation density function from diffusion-weighted MRI data using spherical deconvolution. NeuroImage 2004;23:1176-85.

[42] Tournier J-D, Calamante F, Connelly A. Robust determination of the fibre orientation distribution in diffusion MRI: Non-negativity constrained super-resolved spherical deconvolution. NeuroImage 2007;35:1459-72.

[43] Jeurissen B, Tournier J-D, Dhollander T, Connelly A, Sijbers J. Multi-tissue constrained spherical deconvolution for improved analysis of multi-shell diffusion MRI data. NeuroImage 2014;103:411-26.

[44] Desikan RS, Ségonne F, Fischl B, Quinn BT, Dickerson BC, Blacker D, et al. An automated labeling system for subdividing the human cerebral cortex on MRI scans into gyral based regions of interest. NeuroImage 2006;31:968-80.

[45] Fischl B. FreeSurfer. Neuroimage 2012;62:774-81.

[46] Sporns O, Tononi G, Kötter R. The Human Connectome: A Structural Description of the Human Brain. PLoS Comput Biol 2005;1:e42.

[47] Hagmann P. From Diffusion MRI To Brain Connectomics. PhD Thesis. ÉCOLE POLYTECHNIQUE FÉDÉRALE DE LAUSANNE, 2005. https://doi.org/10.5075/epfl-thesis-3230.

[48] Calamante F, Tournier J-D, Jackson GD, Connelly A. Track-density imaging (TDI): Super-resolution white matter imaging using whole-brain track-density mapping. NeuroImage 2010;53:1233-43.

[49] Stadlbauer A, Buchfelder M, Salomonowitz E, Ganslandt O. Fiber Density Mapping of Gliomas: Histopathologic Evaluation of a Diffusion-Tensor Imaging Data Processing Method. Radiology 2010;257:846-53. https://doi.org/10. $\underline{1148 / \text { radiol.10100343. }}$.

[50] Bozzali M, Parker GJM, Serra L, Embleton K, Gili T, Perri R, et al. Anatomical connectivity mapping: A new tool to assess brain disconnection in Alzheimer's disease. NeuroImage 2011;54:2045-51.

[51] Maier-Hein KH, Neher PF, Houde J-C, Côté M-A, Garyfallidis E, Zhong J, et al. The challenge of mapping the human connectome based on diffusion tractography. Nature Communications 2017;8:1349.

[52] Raffelt DA, Tournier J-D, Smith RE, Vaughan DN, Jackson G, Ridgway GR, et al. Investigating white matter fibre density and morphology using fixel-based analysis. NeuroImage 2017;144:58-73.

[53] Hagmann P, Cammoun L, Gigandet X, Meuli R, Honey CJ, Wedeen VJ, et al. Mapping the Structural Core of Human Cerebral Cortex. PLoS Biology 2008;6:e159.

[54] Zalesky A, Sarwar T, Ramamohanarao K. A cautionary note on the use of SIFT in pathological connectomes. Magnetic Resonance in Medicine 2020;83:791-4. https://doi.org/10.1002/mrm.28037.

[55] Smith RE, Calamante F, Connelly A. Notes on "A cautionary note on the use of SIFT in pathological connectomes". Magnetic Resonance in Medicine 2020;84:2303-7. https://doi.org/10.1002/mrm.28266.

[56] Sherbondy A, Rowe M, Alexander D. MicroTrack: An Algorithm for Concurrent Projectome and Microstructure Estimation. In: Jiang T, Navab N, Pluim J, Viergever M, editors. Medical Image Computing and Computer-Assisted Intervention, vol. 6361, Springer Berlin / Heidelberg; 2010, pp. 183-90.

[57] Lemkaddem A, Skiöldebrand D, Dal Palú A, Thiran J-P, Daducci A. Global tractography with embedded anatomical priors for quantitative connectivity analysis. Frontiers in Neurology 2014;5:232.

[58] Yeh C-H, Smith RE, Dhollander T, Calamante F, Connelly A. Connectomes from streamlines tractography: Assigning streamlines to brain parcellations is not trivial but highly consequential. NeuroImage 2019;199:160-71. https://doi.org/10.1016/j.neuroimage.2019.05.005.

[59] Yeh C-H, Smith RE, Dhollander T, Connelly A. Mesh-based anatomically-constrained tractography for effective tracking termination and structural connectome construction. In:. Proceedings of the ISMRM, 2017, p. 0058.

[60] Smith R, Skoch A, Bajada C, Caspers S, Connelly A. Hybrid Surface-Volume Segmentation for improved Anatomically-Constrained Tractography. In:. Organisation for Human Brain Mapping, 2020, p. 1034.

[61] Yeh C-H, Smith RE, Liang X, Calamante F, Connelly A. Correction for diffusion MRI fibre tracking biases: The consequences for structural connectomic metrics. NeuroImage 2016;142:150-62. 
[62] Morris DM, Embleton KV, Parker GJM. Probabilistic fibre tracking: Differentiation of connections from chance events. NeuroImage 2008;42:1329-39.

[63] Tournier J-D, Smith R, Raffelt D, Tabbara R, Dhollander T, Pietsch M, et al. MRtrix3: A fast, flexible and open software framework for medical image processing and visualisation. NeuroImage 2019;202:116137. https: //doi.org/10.1016/j.neuroimage.2019.116137. 